\title{
Algorithms for cautious reasoning in games*
}

\author{
Geir B. Asheim ${ }^{a} \quad$ Andrés Perea $^{b}$
}

26 April 2019

\begin{abstract}
We provide comparable algorithms for the Dekel-Fudenberg procedure, iterated admissibility, proper rationalizability and full permissibility by means of the notions of likelihood orderings and preference restrictions. The algorithms model reasoning processes whereby each player's preferences over his own strategies are completed by eliminating likelihood orderings.

We apply the algorithms for comparing iterated admissibility, proper rationalizability and full permissibility, and provide a sufficient condition under which iterated admissibility does not rule out properly rationalizable strategies. We also use the algorithms to examine an economically relevant strategic situation, namely a bilateral commitment bargaining game. Finally, we discuss the relevance of our algorithms for epistemic analysis.
\end{abstract}

JEL Classification No.: C72, C78.

Keywords: Non-cooperative games, proper rationalizability, iterated admissibility, bargaining.

${ }^{*}$ We thank José Carlos Alcantud, Walter Bossert, Martin Dufwenberg, Tore Ellingsen, Xiao Luo, Hervé Moulin and Christian Traeger for useful suggestions and two anonymous referees for detailed and constructive comments. We also appreciate helpful comments received from participants at seminars in Ningbo, Paris and Singapore, and at conferences in Maastricht (SED) and Faro (SAET). Asheim gratefully acknowledges the hospitality of the Department of Economics at University of California, Santa Barbara, and CIREQ (Centre interuniversitaire de recherche en économie quantitative - Montréal), and support from l'IMéRA (Institut d'études avancées d'Aix-Marseille Université).

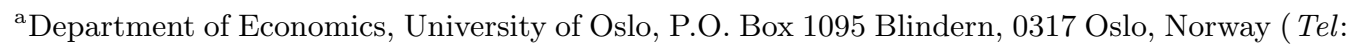
+47 45505 136, Email: g.b.asheim@econ.uio.no).

${ }^{\mathrm{b}}$ Department of Quantitative Economics, Maastricht University, P.O. Box 616, 6200 MD Maastricht, The Netherlands (Tel: +31 4338839 22, Email: a.perea@maastrichtuniversity.nl). 


\section{Introduction}

In non-cooperative game theory, a player is cautious if he takes into account all opponent strategies, also strategies that seem very unlikely to be chosen by the opponents. Caution implies that a player prefers one strategy to another if the former weakly dominates the latter. This concept can be modeled by allowing a player $i$ to deem some strategy $s_{j}$ of an opponent $j$ infinitely more likely than some other strategy $s_{j}^{\prime}$, while still taking $s_{j}^{\prime}$ into account (Blume et al., 1991a). What reasoning in a strategic game is consistent with, not only players being cautious, but also believing that the opponents are rational and cautious, believing that the opponents believe that their opponents are rational and cautious, and so on?

Various concepts in the literature provide different answers to this question. Still, there is a common idea underlying each of these concepts, namely that player $i$ should deem a strategy $s_{j}$ of opponent $j$ infinitely more likely than strategy $s_{j}^{\prime}$ whenever player $i$ considers $s_{j}$ a "better choice" for opponent $j$ than $s_{j}^{\prime}$. The question then remains what we should mean by a "better choice".

As an illustration, consider the following economic example (which is the Spy game of Perea, 2012, p. 262, but with another motivating story). An entrant (firm 1) and an incumbent (firm 2) must decide which type of good to bring to the market: $x, y$ or $z$. The entrant expects a revenue of 3 as long as it produces a good different from the incumbent, and a revenue of 2 if it produces the same good. Its production costs for each of the goods is 2 . The incumbent expects, for every production choice, a revenue of 3 . The only exception is when the goods $x$ and $z$ are both brought on the market. Since these goods are complementary, the incumbent expects a revenue of 6 in this case. The incumbent has produced good $x$ in the past, which would therefore have the lowest costs (normalized to 0). Producing goods $y$ and $z$ would cost the incumbent 1 and 2, respectively, since good $y$ is more similar to $x$ than $z$ is. The profits for both firms can be found in Figure 0, where the choice of firm 1 is indicated in upper case, to differentiate from the choice of firm 2 in lower case.

Note that for firm 2, production choice $y$ can never be rational as $x$ weakly (and strictly) dominates $y$, whereas $x$ and $z$ might as they are not even weakly dominated. One could therefore argue that $x$ and $z$ are better choices for firm 2 than $y$, and hence firm 1 should deem $x$ and $z$ infinitely more likely than $y$. But then, since $Y$ weakly dominates both $X$ and $Z$ on the subset $\{x, z\}$, firm 1's unique rational choice would be to implement production plan $Y$. The line of argument we have followed here 


\begin{tabular}{c|c|c|c|}
\multicolumn{1}{c}{$x$} & \multicolumn{1}{c}{$y$} & \multicolumn{1}{c}{$z$} \\
\cline { 2 - 4 }$X$ & 0,3 & 1,2 & 1,4 \\
\hline$Y$ & 1,3 & 0,2 & 1,1 \\
\cline { 2 - 4 }$Z$ & 1,6 & 1,2 & 0,1 \\
\cline { 2 - 4 } & & &
\end{tabular}

Figure 0: Illustrating iterated admissiblity and proper rationalizability.

corresponds to the procedure of iterated admissibility which iteratedly eliminates all weakly dominated strategies, as it corresponds to the epistemic foundation provided for this procedure by Brandenburger et al. (2008, Theorem 9.1) for any finite number of iterations.

Iterated admissibility is not the only plausible procedure for cautious reasoning, however. Consider again the example above. If firm 2 would indeed believe that firm 1 makes production choice $Y$, which is what iterated admissibility requires, then choice $y$ would actually be better for firm 2 than choice $z$. So given that firm 1 believes that firm 2 believes that firm 1 will do the choice that iterated admissibility requires, one could argue that firm 1 should deem $y$ infinitely more likely than $z$, and not infinitely less likely, as iterated admissibility imposes. Hence, by applying the procedure of iterated admissibility one may along the way impose conditions on beliefs which need not be convincing given the prescriptions that this procedure ends up providing. Such problems with iterated admissibility were already pointed out, discussed and analyzed by Samuelson (1992).

The concept of proper rationalizability (Schuhmacher, 1999; Asheim, 2001) takes a different viewpoint. The key condition is that a player $i$ should deem a strategy $s_{j}$ of opponent $j$ infinitely more likely than strategy $s_{j}^{\prime}$ whenever he believes that opponent $j$, after completing his reasoning process, prefers $s_{j}$ to $s_{j}^{\prime}$. If the beliefs of player $i$ satisfies this condition, we say (following Blume et al., 1991b, Definition 4) that player $i$ respects the preferences of opponent $j$.

To see what difference this approach makes, let us return to the example. It is clear that for firm 2, choice $x$ is better than choice $y$, whereas we cannot say at this stage of the reasoning process that $z$ is better than $y$. Proper rationalizability therefore only requires that firm 1 deems $x$ infinitely more likely than $y$, but does not require that it deems $z$ infinitely more likely than $y$. If firm 1 indeed holds such a belief, then firm 1 prefers $Y$ to $X$ as $Y$ weakly dominates $X$ on both $\{x\}$ and $\{x, z\}$, implying that firm 2 should deem $Y$ infinitely more likely than $X$. But then, firm 2 will, in addition to prefering $x$ to $y$, also prefer $y$ to $z$ as $y$ weakly dominates $z$ on 
both $\{Y\}$ and $\{Y, Z\}$. Hence, firm 1 should deem $x$ infinitely more likely than $y$, and $y$ infinitely more likely than $z$. As a consequence, firm 1 should choose production plan $Z$, and not $Y$ as iterated admissibility requires.

The concept of full permissibility (Asheim and Dufwenberg, 2003a) represents an intermediate position: Based on the observation that $y$ can never be a rational choice for firm 2, firm 1 might (i) deem both $x$ and $z$ infinitely more likely than $y$, (ii) deem $x$ infinitely more likely than both $y$ and $z$, or (iii) deem $z$ infinitely more likely than both $x$ and $y$. In case (i), $X$ and $Z$ are ruled out as rational choices for firm 1, leading firm 2 to deem $Y$ infinitely more likely than both $X$ and $Z$. In case (ii), only $X$ is ruled out as a rational choice for firm 1, leading firm 2 to deem both $Y$ and $Z$ infinitely more likely than $X$. Lastly, in case (iii), only $Z$ is ruled out as a rational choice for firm 1, leading firm 2 to deem both $X$ and $Y$ infinitely more likely than $Z$. However, in neither of these cases, can $z$ be the sole rational choice for firm 2 , ruling out case (iii) where firm 1 deems $z$ infinitely more likely than both $x$ and $y$. This precludes that firm 2 deems both $X$ and $Y$ infinitely more likely than $Z$. But then, $x$ and $z$ cannot both be rational choices for firm 2, ruling out case (i) and implying that firm 1 , in line with case (ii), deems $x$ infinitely more likely than both $y$ and $z$. Thus, only $X$ is ruled out as a rational choice for firm 1, in which case firm 2 deems both $Y$ and $Z$ infinitely more likely than $X$. Thereby, full permissibility promotes the choice sets $\{Y, Z\}$ and $\{x\}$ for firms 1 and 2 respectively.

All three concepts, iterated admissibility, proper rationalizability and full permissibility, are reasonable concepts with their own intuitive appeal, but may lead to completely different reasoning and choices as we have seen. It therefore seems worthwhile to investigate their differences and similarities in some more detail, and this is exactly what this paper seeks to accomplish.

A number of contributions, starting with Brandenburger (1992) and Börgers (1994), have shown that the Dekel-Fudenberg procedure (Dekel and Fudenberg, 1990), where one round of elimination of weakly dominated strategies is followed by iterated elimination of strictly dominated strategies, provides a robust answer to the question we posed initially, in the sense that the eliminated strategies are definitely incompatible with iterated beliefs of the event that all players are rational and cautious. Hence, surviving the Dekel-Fudenberg procedure, and thus being permissible in the terminology of Brandenburger (1992), is a necessary condition. However, the concepts of iterated admissibility, proper rationalizability and full permissibility might rule out more strategies. This is indeed the case in the game of Figure 0, 
where the Dekel-Fudenberg procedure eliminates only $y$ for firm 2, leading to $X, Y$ and $Z$ being permissible for firm 1 and $x$ and $z$ being permissible for firm 2 .

Permissibility, iterated admissibility and full permissibility are all defined in terms of algorithms. While epistemic foundations for the former and latter were provided quickly (Brandenburger, 1992; Börgers, 1994; Asheim and Dufwenberg, 2003a), half a century elapsed between the introduction of iterated admissibility in the 1950s and the establishment of epistemic foundations for this procedure by Brandenburger et al. (2008) and later contributions (cf. footnote 2).

The case of proper rationalizability is different. This concept was defined by Schuhmacher (1999) and Asheim (2001) by means of epistemic conditions. Schuhmacher defines, for every $\varepsilon>0$, the $\varepsilon$-proper trembling condition, which states that if a player prefers one pure strategy over another, then the probability he assigns to the latter strategy should be at most $\varepsilon$ times the probability he assigns to the former. Proper rationalizability is obtained by imposing common belief of the $\varepsilon$-proper trembling condition, and then letting $\varepsilon$ tend to zero. Schuhmacher (1999) provides an algorithm, iteratively proper trembling, which generates for a given $\varepsilon>0$ the set of mixed strategy profiles that can be chosen under common belief of the $\varepsilon$-proper trembling condition. However, this procedure does not yield the set of properly rationalizable strategies directly, as we must still let $\varepsilon$ go to zero, and see which strategies survive in the limit. Only later has Perea (2011) provided an algorithm that directly computes the set of properly rationalizable strategies.

The present paper presents algorithms for permissibility, iterated admissibility and full permissibility that are comparable to Perea (2011)'s algorithm for proper rationalizability. In all cases, the reasoning process leads to preferences that become more complete with additional levels of reasoning. If a player is cautious but does not reason about the behavior of his opponents, then he can only rank strategies that weakly dominate each other on the whole set of opponent strategy profiles. However, by realizing that some opponent strategies are "better choices" than others, the player might also be able to rank strategies that weakly dominate each other on strict subsets of opponent strategy profiles, as illustrated by the game of Figure 0. Considering that opponents also engage in similar kinds of reasoning reduces his strategic uncertainty even more and leads to further completion of preferences, and so on.

It is a main observation of the present paper that, in the case all four concepts considered, the reasoning process can be captured by the key notions introduced by Perea (2011): likelihood orderings and preference restrictions. More specifically, 
the completion of preferences with increasing levels of reasoning corresponds to elimination of likelihood orderings, while the reasoning about opponent behavior is captured by sets of preference restrictions derived from their sets of remaining likelihood orderings.

Section 2 defines the notions of likelihood orderings and preference restrictions and explores how a player's set of likelihood orderings corresponds to (possibly incomplete) preferences over the player's own strategies. Section 3 introduces algorithms that iteratedly eliminate likelihood orderings, for the concepts of permissibility, iterated admissibility and full permissibility. These algorithms are thus comparable with the one for proper rationalizability. Section 4 then puts these algorithms to use. In particular, we offer examples further illuminating the differences between iterated admissibility, proper rationalizability and full permissibility. Moreover, we provide a sufficient condition under which iterated admissibility does not rule out properly rationalizable strategies. Finally, we use the algorithms to examine an economically relevant strategic situation, namely a bilateral commitment bargaining game which has been analyzed by Ellingsen and Miettinen (2008). Section 5 offers concluding remarks, in particular by discussing the relevance of our algorithms for epistemic analysis. An appendix contains all proofs.

\section{Likelihood orderings and preference restrictions}

Consider a finite strategic game $G=\left(S_{i}, u_{i}\right)_{i \in I}$ where $I$ is a finite set of players and where, for $i \in I$, the finite set $S_{i}$ denotes player $i$ 's set of strategies and $u_{i}$ : $\prod_{j \in I} S_{j} \rightarrow \mathbb{R}$ denotes player $i$ 's utility function. Write $S_{-i}:=\prod_{j \neq i} S_{j}$ and $S_{-i, j}:=$ $\prod_{j^{\prime} \neq i, j} S_{j^{\prime}}$. As usual, we extend $u_{i}$ to objective probability distributions (mixed strategies $) \mu_{i} \in \Delta\left(S_{i}\right)$ over player $i$ 's strategies, writing $u_{i}\left(\mu_{i}, s_{-i}\right)$ for the resulting objective expected utility, and to subjective probability distributions $\lambda_{i} \in \Delta\left(S_{-i}\right)$ over the opponent's strategy profiles, writing $u_{i}\left(s_{i}, \lambda_{i}\right)$ for the resulting subjective expected utility.

For all mixed strategies $\mu_{i}^{\prime}, \mu_{i}^{\prime \prime} \in \Delta\left(S_{i}\right)$, say that $\mu_{i}^{\prime}$ strictly dominates $\mu_{i}^{\prime \prime}$ on a subset $S_{-i}^{\prime} \subseteq S_{-i}$ of opponent strategy profiles if $u_{i}\left(\mu_{i}^{\prime}, s_{-i}\right)>u_{i}\left(\mu_{i}^{\prime \prime}, s_{-i}\right)$ for every $s_{-i} \in S_{-i}^{\prime}$. Similarly, say that $\mu_{i}^{\prime}$ weakly dominates $\mu_{i}^{\prime \prime}$ on $S_{-i}^{\prime}$ if $u_{i}\left(\mu_{i}^{\prime}, s_{-i}\right) \geq$ $u_{i}\left(\mu_{i}^{\prime \prime}, s_{-i}\right)$ for every $s_{-i} \in S_{-i}^{\prime}$, with strict inequality for some $s_{-i}^{\prime} \in S_{-i}^{\prime}$.

Each player $i$ 's preferences over his own strategies are determined by $u_{i}$ and a non-empty set $\mathcal{L}_{i}$ of likelihood orderings on $S_{-i}$. The notion of a likelihood ordering 
was used by Perea (2011) and is one of the two key definitions for our algorithms.

Definition 1 (Likelihood ordering) A likelihood ordering for player $i$ on $S_{-i}$ is an ordered partition $L_{i}=\left(L_{i}^{1}, L_{i}^{2}, \ldots, L_{i}^{K}\right)$ of $S_{-i}$.

Let $\mathcal{L}_{i}^{*}$ denote the set of all likelihood orderings on $S_{-i}$. Note that $\mathcal{L}_{i}^{*}$ is finite. Let $\tilde{\mathcal{L}}_{i}^{*}\left(\subseteq \mathcal{L}_{i}^{*}\right)$ denote the set of all likelihood orderings on $S_{-i}$ which are either trivial (so that $K=1$ and $L_{i}=\left(L_{i}^{1}\right)=\left(S_{-i}\right)$ ) or partition $S_{-i}$ into a non-empty proper subset $S_{-i}^{\prime}$ and its complement (so that $K=2$ and $L_{i}=\left(L_{i}^{1}, L_{i}^{2}\right)=\left(S_{-i}^{\prime}, S_{-i} \backslash S_{-i}^{\prime}\right)$ ).

Assume that player $i$ has a non-empty set $\mathcal{L}_{i}$ of likelihood orderings. Then, for all mixed strategies $\mu_{i}^{\prime}, \mu_{i}^{\prime \prime} \in \Delta\left(S_{i}\right)$, player $i$ prefers $\mu_{i}^{\prime}$ to $\mu_{i}^{\prime \prime}$ under $\mathcal{L}_{i}$ (written $\left.\mu_{i}^{\prime} \succ_{i}^{\mathcal{L}_{i}} \mu_{i}^{\prime \prime}\right)$ if, for all $L_{i}=\left(L_{i}^{1}, L_{i}^{2}, \ldots, L_{i}^{K}\right) \in \mathcal{L}_{i}$, there exists $k \in\{1, \ldots, K\}$ such that $\mu_{i}^{\prime}$ weakly dominates $\mu_{i}^{\prime \prime}$ on $L_{i}^{1} \cup \cdots \cup L_{i}^{k}$, and player $i$ deems $\mu_{i}^{\prime}$ equally as good as $\mu_{i}^{\prime \prime}$ (written $\left.\mu_{i}^{\prime} \sim_{i}^{\mathcal{L}_{i}} \mu_{i}^{\prime \prime}\right)$ if $u_{i}\left(\mu_{i}^{\prime}, s_{-i}\right)=u_{i}\left(\mu_{i}^{\prime \prime}, s_{-i}\right)$ for every $s_{-i} \in S_{-i}$. It holds for any non-empty set $\mathcal{L}_{i}$ of likelihood orderings that $\mu_{i}^{\prime} \succ_{i}^{\mathcal{L}_{i}} \mu_{i}^{\prime \prime}$ if $\mu_{i}^{\prime}$ weakly dominates $\mu_{i}^{\prime \prime}$ on $S_{-i}$ since, for all $L_{i}=\left(L_{i}^{1}, L_{i}^{2}, \ldots, L_{i}^{K}\right) \in \mathcal{L}_{i}, L_{i}^{1} \cup \cdots \cup L_{i}^{K}=S_{-i}$.

Write $\mu_{i}^{\prime} \succsim_{i}^{\mathcal{L}_{i}} \mu_{i}^{\prime \prime}$ if $\mu_{i}^{\prime} \succ_{i}^{\mathcal{L}_{i}} \mu_{i}^{\prime \prime}$ or $\mu_{i}^{\prime} \sim_{i}^{\mathcal{L}_{i}} \mu_{i}^{\prime \prime}$.

Lemma 1 Let $\mathcal{L}_{i}$ be player $i$ 's non-empty set of likelihood orderings. The $\sim_{i}^{\mathcal{L}_{i}}$ and $\succsim_{i}^{\mathcal{L}_{i}}$ relations are reflexive and transitive, and the $\succ_{i}^{\mathcal{L}_{i}}$ relation is irreflexive and transitive. Furthermore, the $\sim_{i}^{\mathcal{L}_{i}}$ and $\succ_{i}^{\mathcal{L}_{i}}$ relations satisfy objective independence. ${ }^{1}$

Remark 1 While the $\succsim_{i}^{\mathcal{L}_{i}}$ relation is always reflexive and transitive, it need not be complete. To see this, let $I=\{1,2\}, S_{1}=\left\{s_{1}^{\prime}, s_{1}^{\prime \prime}\right\}, S_{2}=\left\{s_{2}^{\prime}, s_{2}^{\prime \prime}\right\}, u_{1}\left(s_{1}^{\prime}, s_{2}^{\prime}\right)=1$, $u_{1}\left(s_{1}^{\prime \prime}, s_{2}^{\prime}\right)=0, u_{1}\left(s_{1}^{\prime}, s_{2}^{\prime \prime}\right)=0$, and $u_{1}\left(s_{1}^{\prime \prime}, s_{2}^{\prime \prime}\right)=1$. Then $s_{1}^{\prime} \nsucc_{1}^{\mathcal{L}_{1}} s_{1}^{\prime \prime}, s_{1}^{\prime} \nsim_{1}^{\mathcal{L}_{1}} s_{1}^{\prime \prime}$ and $s_{1}^{\prime} \nprec_{1}^{\mathcal{L}_{1}} s_{1}^{\prime \prime}$ if $\mathcal{L}_{1}=\mathcal{L}_{1}^{*}=\left\{\left(\left\{s_{2}^{\prime}\right\},\left\{s_{2}^{\prime \prime}\right\}\right),\left(\left\{s_{2}^{\prime \prime}\right\},\left\{s_{2}^{\prime}\right\}\right),\left(S_{2}\right)\right\}$. However, $s_{1}^{\prime} \succ_{1}^{\mathcal{L}_{1}} s_{1}^{\prime \prime}$ if $\mathcal{L}_{1}=\left\{\left(\left\{s_{2}^{\prime}\right\},\left\{s_{2}^{\prime \prime}\right\}\right)\right\}$.

These preferences can be characterized by means of the lexicographic probability system (LPS) concept and related to the infinitely more likely relation, both due to Blume et al. (1991a).

A lexicographic probability system (LPS) on $S_{-i}$ consists of a finite sequence of subjective probability distributions, $\lambda_{i}=\left(\lambda_{i}^{1}, \lambda_{i}^{2}, \ldots, \lambda_{i}^{K}\right)$, where for each $k \in$ $\{1, \ldots, K\}, \lambda_{i}^{k} \in \Delta\left(S_{-i}\right)$. Consider two mixed strategies $\mu_{i}^{\prime}, \mu_{i}^{\prime \prime} \in \Delta\left(S_{i}\right)$. The LPS $\lambda_{i}$ ranks $\mu_{i}^{\prime}$ above $\mu_{i}^{\prime \prime}$ if there exists $k \in\{1, \ldots, K\}$ such that (i) $u_{i}\left(\mu_{i}^{\prime}, \lambda_{i}^{k}\right)>u_{i}\left(\mu_{i}^{\prime \prime}, \lambda_{i}^{k}\right)$

\footnotetext{
${ }^{1}$ Say that a binary relation $\sim(\succ)$ satisfies objective independence if, for all $\mu_{i}^{\prime}, \mu_{i}^{\prime \prime}, \mu_{i}^{\prime \prime \prime} \in \Delta\left(S_{i}\right)$ and $\gamma \in(0,1), \mu_{i}^{\prime} \sim(\succ) \mu_{i}^{\prime \prime}$ if and only if $\gamma \mu_{i}^{\prime}+(1-\gamma) \mu_{i}^{\prime \prime \prime} \sim(\succ) \gamma \mu_{i}^{\prime \prime}+(1-\gamma) \mu_{i}^{\prime \prime \prime}$.
} 
and (ii) $u_{i}\left(\mu_{i}^{\prime}, \lambda_{i}^{\ell}\right)=u_{i}\left(\mu_{i}^{\prime \prime}, \lambda_{i}^{\ell}\right)$ for all $\ell \in\{1, \ldots, k-1\}$. The LPS $\lambda_{i}$ deems $\mu_{i}^{\prime}$ indifferent to $\mu_{i}^{\prime \prime}$ if $u_{i}\left(\mu_{i}^{\prime}, \lambda_{i}^{k}\right)=u_{i}\left(\mu_{i}^{\prime \prime}, \lambda_{i}^{k}\right)$ for all $k \in\{1, \ldots, K\}$. For given LPS, the ranking-above-or-deeming-indifferent-to relation is complete and transitive. The LPS $\lambda_{i}=\left(\lambda_{i}^{1}, \ldots, \lambda_{i}^{K}\right)$ has full support on $S_{-i}$ if $\operatorname{supp} \lambda_{i}^{1} \cup \cdots \cup \operatorname{supp} \lambda_{i}^{K}=S_{-i}$. Say that the LPS $\lambda_{i}=\left(\lambda_{i}^{1}, \ldots, \lambda_{i}^{K}\right)$ is consistent with the likelihood ordering $L_{i}=$ $\left(L_{i}^{1}, L_{i}^{2}, \ldots, L_{i}^{K}\right)$ if, for all $k \in\{1, \ldots, K\}, \operatorname{supp} \lambda_{i}^{1} \cup \cdots \cup \operatorname{supp} \lambda_{i}^{k}=L_{i}^{1} \cup \cdots \cup L_{i}^{k}$. An LPS $\lambda_{i}$ has full support on $S_{-i}$ if consistent with a likelihood ordering $L_{i}$.

Lemma 2 Let $\mathcal{L}_{i}$ be player i's non-empty set of likelihood orderings. For all mixed strategies $\mu_{i}^{\prime}, \mu_{i}^{\prime \prime} \in \Delta\left(S_{i}\right), \mu_{i}^{\prime} \succ_{i}^{\mathcal{L}_{i}} \mu_{i}^{\prime \prime}$ if and only if, for all $L_{i} \in \mathcal{L}_{i}$, every LPS $\lambda_{i}$ consistent with $L_{i}$ ranks $\mu_{i}^{\prime}$ above $\mu_{i}^{\prime \prime}$, and $\mu_{i}^{\prime} \sim_{i}^{\mathcal{L}_{i}} \mu_{i}^{\prime \prime}$ if and only if, for all $L_{i} \in \mathcal{L}_{i}$, every LPS $\lambda_{i}$ consistent with $L_{i}$ deems $\mu_{i}^{\prime}$ indifferent to $\mu_{i}^{\prime \prime}$.

Assume that player $i$ has a non-empty set $\mathcal{L}_{i}$ of likelihood orderings. Consider two pure opponent strategy profiles $s_{-i}^{\prime}, s_{-i}^{\prime \prime} \in S_{-i}$. Player $i$ deems $s_{-i}^{\prime}$ infinitely more likely than $s_{-i}^{\prime \prime}$ under $\mathcal{L}_{i}$ (written $s_{-i}^{\prime} \gg_{i}^{\mathcal{L}_{i}} s_{-i}^{\prime \prime}$ ) if, for all $L_{i}=\left(L_{i}^{1}, L_{i}^{2}, \ldots, L_{i}^{K}\right) \in \mathcal{L}_{i}$, there exists $k \in\{1, \ldots, K\}$ such that $s_{-i}^{\prime} \in L_{i}^{1} \cup \cdots \cup L_{i}^{k}$ and $s_{-i}^{\prime \prime} \notin L_{i}^{1} \cup \cdots \cup L_{i}^{k}$. Furthermore, for each $j \neq i$, player $i$ deems $s_{j}^{\prime}$ infinitely more likely than $s_{j}^{\prime \prime}$ under $\mathcal{L}_{i}$ (written $s_{j}^{\prime} \gg_{i}^{\mathcal{L}_{i}} s_{j}^{\prime \prime}$ ) if there exists some $s_{-i}^{\prime} \in\left\{s_{j}^{\prime}\right\} \times S_{-i, j}$ such that $s_{-i}^{\prime} \gg_{i}^{\mathcal{L}_{i}} s_{-i}^{\prime \prime}$ for all $s_{-i}^{\prime \prime} \in\left\{s_{j}^{\prime \prime}\right\} \times S_{-i, j}$. In the following remark we observe that this definition is consistent with the concept as defined by Blume et al. (1991a, Definition 5.1).

Remark 2 Let $\mathcal{L}_{i}$ be player i's non-empty set of likelihood orderings. For all mixed strategies $\mu_{i}^{\prime}, \mu_{i}^{\prime \prime} \in \Delta\left(S_{i}\right)$ and all pure opponent strategy profiles $s_{-i}^{\prime}, s_{-i}^{\prime \prime} \in S_{-i}$, if $s_{-i}^{\prime} \gg_{i}^{\mathcal{L}_{i}} s_{-i}^{\prime \prime}$, then $\mu_{i}^{\prime} \succ_{i}^{\mathcal{L}_{i}} \mu_{i}^{\prime \prime}$ whenever $\mu_{i}^{\prime}, \mu_{i}^{\prime \prime} \in \Delta\left(S_{i}\right)$ satisfy $u_{i}\left(\mu_{i}^{\prime}, s_{-i}^{\prime}\right)>$ $u_{i}\left(\mu_{i}^{\prime \prime}, s_{-i}^{\prime}\right)$ and, for all $s_{-i} \in S_{-i} \backslash\left\{s_{-i}^{\prime}, s_{-i}^{\prime \prime}\right\}, u_{i}\left(\mu_{i}^{\prime}, s_{-i}\right)=u_{i}\left(\mu_{i}^{\prime \prime}, s_{-i}\right)$.

The converse of the implication in Remark 2 cannot be shown as we consider a particular game $G$ with a particular utility function $u_{i}$ for player $i$.

We are now ready to introduce the other key notion of our algorithms, also taken from Perea (2011).

Definition 2 (Preference restriction) A preference restriction for player $i$ on $S_{i}$ is a pair $\left(s_{i}, A_{i}\right)$, where $s_{i} \in S_{i}$ and $A_{i}$ is a nonempty subset of $S_{i}$.

Let $R_{i}$ denote a set of preference restrictions for $i$, and let $\mathcal{R}_{i}^{*}$ denote the collection of all sets of preference restrictions for $i$. 
For any non-empty set $\mathcal{L}_{i}$ of likelihood orderings, let $R_{i}\left(\mathcal{L}_{i}\right)$ denote the set of preference restrictions derived from $\mathcal{L}_{i}$, where $R_{i}\left(\mathcal{L}_{i}\right)$ is defined as follows:

$$
R_{i}\left(\mathcal{L}_{i}\right):=\left\{\left(s_{i}, A_{i}\right) \in S_{i} \times 2^{S_{i}} \mid \exists \mu_{i} \in \Delta\left(A_{i}\right) \text { such that } \mu_{i} \succ_{i}^{\mathcal{L}_{i}} s_{i}\right\} .
$$

Hence, the interpretation of a preference restriction $\left(s_{i}, A_{i}\right)$ in the set $R_{i}\left(\mathcal{L}_{i}\right)$ is that player $i$ prefers some mixed strategy in $\Delta\left(A_{i}\right)$ to $s_{i}$ under $\mathcal{L}_{i}$. It follows that $R_{i}\left(\mathcal{L}_{i}^{\prime}\right) \cap R_{i}\left(\mathcal{L}_{i}^{\prime \prime}\right)=R_{i}\left(\mathcal{L}_{i}^{\prime} \cup \mathcal{L}_{i}^{\prime \prime}\right)$ for every $\mathcal{L}_{i}^{\prime}, \mathcal{L}_{i}^{\prime \prime} \subseteq \mathcal{L}_{i}^{*}$. In particular, $R_{i}\left(\mathcal{L}_{i}^{\prime}\right) \supseteq R_{i}\left(\mathcal{L}_{i}^{\prime \prime}\right)$ whenever $\mathcal{L}_{i}^{\prime} \subseteq \mathcal{L}_{i}^{\prime \prime}$. Abuse notation slightly by writing $R_{i}\left(L_{i}\right)$ instead of $R_{i}\left(\mathcal{L}_{i}\right)$ if $\mathcal{L}_{i}=\left\{L_{i}\right\}$ is a singleton set of likelihood orderings.

Lemma 3 Let $\mathcal{L}_{i}$ be player $i$ 's non-empty set of likelihood orderings. If $\left(s_{i}, A_{i}\right) \in$ $R_{i}\left(\mathcal{L}_{i}\right)$, then there exists $A_{i}^{\prime} \subseteq S_{i} \backslash\left\{s_{i}\right\}$ such that $\left(s_{i}, A_{i}^{\prime}\right) \in R_{i}\left(\mathcal{L}_{i}\right)$.

Remark 3 The set $R_{i}\left(\mathcal{L}_{i}\right)$ of derived preference restrictions need not be non-empty. To see this, consider the example of Remark 1 , where $s_{1}^{\prime} \nsucc_{1}^{\mathcal{L}_{1}} s_{1}^{\prime \prime}, s_{1}^{\prime} \nprec_{1}^{\mathcal{L}_{1}} s_{1}^{\prime \prime}$ and $R_{1}\left(\mathcal{L}_{1}\right)=\emptyset$ if $\mathcal{L}_{1}=\mathcal{L}_{1}^{*}=\left\{\left(\left\{s_{2}^{\prime}\right\},\left\{s_{2}^{\prime \prime}\right\}\right),\left(\left\{s_{2}^{\prime \prime}\right\},\left\{s_{2}^{\prime}\right\}\right),\left(S_{2}\right)\right\}$. However, $s_{1}^{\prime} \succ_{1}^{\mathcal{L}_{1}} s_{1}^{\prime \prime}$ and $R_{1}\left(\mathcal{L}_{1}\right)=\left\{\left(s_{1}^{\prime \prime},\left\{s_{1}^{\prime}\right\}\right),\left(s_{1}^{\prime \prime}, S_{1}\right)\right\}$ if $\mathcal{L}_{1}=\left\{\left(\left\{s_{2}^{\prime}\right\},\left\{s_{2}^{\prime \prime}\right\}\right)\right\}$.

Assume that player $i$ has the (possibly empty) set, $R_{i}$, of preference restrictions. Define player $i$ 's choice set $C_{i}\left(R_{i}\right)$ as follows:

$$
C_{i}\left(R_{i}\right):=\left\{s_{i} \in S_{i} \mid \nexists A_{i} \subseteq S_{i} \text { with }\left(s_{i}, A_{i}\right) \in R_{i}\right\} .
$$

It follows that $C_{i}\left(R_{i}^{\prime}\right) \cap C_{i}\left(R_{i}^{\prime \prime}\right)=C_{i}\left(R_{i}^{\prime} \cup R_{i}^{\prime \prime}\right)$ for every $R_{i}^{\prime}, R_{i}^{\prime \prime} \in \mathcal{R}_{i}^{*}$. In particular, $C_{i}\left(R_{i}^{\prime}\right) \supseteq C_{i}\left(R_{i}^{\prime \prime}\right)$ whenever $R_{i}^{\prime} \subseteq R_{i}^{\prime \prime}$. Clearly, $C_{i}(\emptyset)=S_{i}$. The following result yields a justification for the term 'choice set'.

Lemma 4 Let $\mathcal{L}_{i}$ be player $i$ 's non-empty set of likelihood orderings. Then

$$
C_{i}\left(R_{i}\left(\mathcal{L}_{i}\right)\right)=\left\{s_{i} \in S_{i} \mid \nexists \mu_{i} \in \Delta\left(S_{i}\right) \text { such that } \mu_{i} \succ_{i}^{\mathcal{L}_{i}} s_{i}\right\} \text {. }
$$

Furthermore, $C_{i}\left(R_{i}\left(\mathcal{L}_{i}\right)\right) \neq \emptyset$.

Let $\mathcal{R}_{-i}^{*}:=\prod_{j \neq i} \mathcal{R}_{i}^{*}$ denote the collection of all vectors of sets of preference restrictions for $i$ 's opponents. If $R_{-i} \in \mathcal{R}_{-i}^{*}$, let $C_{-i}\left(R_{-i}\right):=\prod_{j \neq i} C_{j}\left(R_{j}\right)$ denote the Cartesian product of the choice sets of $i$ 's opponents, given the vector of their sets of preference restrictions. The set $C_{-i}\left(R_{-i}\right)$ is the event that $i$ 's opponents are rational when their preferences satisfy the vector $R_{-i}$ of sets of preference restrictions. Let 
$R_{-i}\left(\mathcal{L}_{-i}\right):=\left(R_{j}\left(\mathcal{L}_{j}\right)\right)_{j \neq i}$ denote the vectors of sets of preference restrictions for $i$ 's opponents given their vector $\mathcal{L}_{-i}:=\left(\mathcal{L}_{j}\right)_{j \neq i}$ of non-empty sets of likelihood orderings. Let $\mathcal{L}_{-i} \neq \emptyset$ signify that $\mathcal{L}_{j} \neq \emptyset$ for all $j \neq i$ and let $\mathcal{L}_{-i}^{\prime} \subseteq \mathcal{L}_{-i}^{\prime \prime}$ signify that $\mathcal{L}_{j}^{\prime} \subseteq \mathcal{L}_{j}^{\prime \prime}$ for all $j \neq i$. Let $R_{-i}\left(L_{-i}\right):=\left(R_{j}\left(L_{j}\right)\right)_{j \neq i}$ denote the vectors of sets of preference restrictions for $i$ 's opponents given their vector $L_{-i}=\left(L_{j}\right)_{j \neq i}$ of singleton sets of likelihood orderings. Let $L_{-i} \in \mathcal{L}_{-i}$ signify that $L_{j} \in \mathcal{L}_{j}$ for all $j \neq i$.

Likelihood-orderings can be related to the ordinary belief operator as well as the assumption operator, as proposed by Brandenburger et al. (2008) (and discussed by Asheim and Søvik, 2005, Section 6).

Definition 3 (Believing an event) For a given subset $A_{-i} \subseteq S_{-i}$ of opponent strategy vectors, the likelihood ordering $L_{i}=\left(L_{i}^{1}, L_{i}^{2}, \ldots, L_{i}^{K}\right)$ believes $A_{-i}$ if $L_{i}^{1} \subseteq$ $A_{-i}$.

Likewise, say that player $i$ with non-empty set $\mathcal{L}_{i}$ of likelihood orderings believes $A_{-i}$ if, for all $L_{i} \in \mathcal{L}_{i}, L_{i}$ believes $A_{-i}$. In the special case where $A_{-i}$ is a singleton set, we have that player $i$ believes $A_{-i}$ if and only if the sole strategy profile $a_{-i}$ in $A_{-i}$ satisfies, for every $s_{-i} \in S_{-i} \backslash A_{-i}, a_{-i} \gg_{i}^{\mathcal{L}_{i}} s_{-i}$.

Definition 4 (Assuming an event) For a given subset $A_{-i} \subseteq S_{-i}$ of opponent strategy vectors, the likelihood ordering $L_{i}=\left(L_{i}^{1}, L_{i}^{2}, \ldots, L_{i}^{K}\right)$ assumes $A_{-i}$ if there exists $k \in\{1, \ldots, K\}$ such that $L_{i}^{1} \cup \cdots \cup L_{i}^{k}=A_{-i}$.

Likewise, say that player $i$ with non-empty set $\mathcal{L}_{i}$ of likelihood orderings assumes $A_{-i}$ if, for all $L_{i} \in \mathcal{L}_{i}, L_{i}$ assumes $A_{-i}$. Hence, player $i$ assumes $A_{-i}$ if and only if $A_{-i} \neq \emptyset$ and, for every $s_{-i} \in S_{-i} \backslash A_{-i}, a_{-i} \gg_{i}^{\mathcal{L}_{i}} s_{-i}$ for every $a_{-i} \in A_{-i}$.

Note that if player $i$ assumes an event $A_{-i}$, then the player also believes the event $A_{-i}$ (since clearly, for all $L_{i}=\left(L_{i}^{1}, L_{i}^{2}, \ldots, L_{i}^{K}\right) \in \mathcal{L}_{i}, L_{i}^{1} \subseteq A_{-i}$ if there exists $k \in\{1, \ldots, K\}$ such that $\left.L_{i}^{1} \cup \cdots \cup L_{i}^{k}=A_{-i}\right)$, but not vice versa.

Likelihood-orderings can also be related to respect of preferences as introduced by Blume et al. (1991b).

Definition 5 (Respecting preferences) For a given vector $R_{-i} \in \mathcal{R}_{-i}^{*}$ of sets of preference restrictions, the likelihood ordering $L_{i}=\left(L_{i}^{1}, L_{i}^{2}, \ldots, L_{i}^{K}\right)$ respects $R_{-i}$ if, for all players $j \neq i$, and every preference restriction $\left(s_{j}, A_{j}\right) \in R_{j}$, there exists $k \in\{1, \ldots, K\}$ such that

$$
\left(L_{i}^{1} \cup \cdots \cup L_{i}^{k}\right) \cap\left(A_{j} \times S_{-i, j}\right) \neq \emptyset \text { and }\left(L_{i}^{1} \cup \cdots \cup L_{i}^{k}\right) \cap\left(\left\{s_{j}\right\} \times S_{-i, j}\right)=\emptyset .
$$


Likewise, say that player $i$ with non-empty set $\mathcal{L}_{i}$ of likelihood orderings respects $R_{-i}$ if, for all $L_{i} \in \mathcal{L}_{i}, L_{i}$ respects $R_{-i}$. If player $i$ respects $R_{-i}$ and there exist a player $j$ and a preference restriction $\left(s_{j}, A_{j}\right)$ such that $A_{j} \backslash\left\{s_{j}\right\}$ is a singleton set, then the sole strategy $a_{j}$ in $A_{j} \backslash\left\{s_{j}\right\}$ satisfies $a_{j} \gg_{i}^{\mathcal{L}_{i}} s_{j}$.

It follows that player $i$ believes the rationality of the opponents if the player respects their preferences, but not vice versa. This can be stated formally as follows.

Lemma 5 If player $i$ with non-empty set $\mathcal{L}_{i}$ of likelihood orderings respects the vector $R_{-i} \in \mathcal{R}_{-i}^{*}$ of sets of preference restrictions, then the player also believes the event $C_{-i}\left(R_{-i}\right)$.

Let $\mathcal{L}_{i}^{b}\left(R_{-i}\right)$ denote the greatest set of likelihood orderings for which player $i$ believes the rationality of $i$ 's opponents when the preferences of $i$ 's opponents satisfy the vector $R_{-i}$ of sets of preference restrictions:

$$
\mathcal{L}_{i}^{b}\left(R_{-i}\right):=\left\{L_{i} \in \mathcal{L}_{i}^{*} \mid L_{i} \text { believes } C_{-i}\left(R_{-i}\right)\right\}
$$

Let $\mathcal{L}_{i}^{a}\left(R_{-i}\right)$ denote the greatest set of likelihood orderings for which player $i$ assumes the rationality of $i$ 's opponents when the preferences of $i$ 's opponents satisfy the vector $R_{-i}$ of sets of preference restrictions:

$$
\mathcal{L}_{i}^{a}\left(R_{-i}\right):=\left\{L_{i} \in \mathcal{L}_{i}^{*} \mid L_{i} \text { assumes } C_{-i}\left(R_{-i}\right)\right\}
$$

Finally, let $\mathcal{L}_{i}^{r}\left(R_{-i}\right)$ denote the greatest set of likelihood orderings for which player $i$ respects the vector $R_{-i}$ of opponent sets of preference restrictions:

$$
\mathcal{L}_{i}^{r}\left(R_{-i}\right):=\left\{L_{i} \in \mathcal{L}_{i}^{*} \mid L_{i} \text { respects } R_{-i}\right\}
$$

Note that $\mathcal{L}_{i}^{b}(\emptyset)=\mathcal{L}_{i}^{a}(\emptyset)=\mathcal{L}_{i}^{r}(\emptyset)=\mathcal{L}_{i}^{*}$. We have seen that assumption implies belief, but not vice versa. Moreover, from Lemma 5 we know that respect of preferences implies belief of rationality, but not versa. Hence, we conclude that

$$
\mathcal{L}_{i}^{b}\left(R_{-i}\right) \supseteq \mathcal{L}_{i}^{a}\left(R_{-i}\right) \cup \mathcal{L}_{i}^{r}\left(R_{-i}\right)
$$

for every $R_{-i} \in \mathcal{R}_{-i}^{*}$ with $C_{-i}\left(R_{-i}\right) \neq \emptyset$. Since the belief operator satisfies conjunction and monotonicity, the properties of the choice correspondence $C_{i}(\cdot)$ imply

$$
\mathcal{L}_{i}^{b}\left(R_{-i}^{\prime}\right) \cap \mathcal{L}_{i}^{b}\left(R_{-i}^{\prime \prime}\right)=\mathcal{L}_{i}^{b}\left(R_{-i}^{\prime} \cup R_{-i}^{\prime \prime}\right)
$$


for every $R_{-i}^{\prime}, R_{-i}^{\prime \prime} \in \mathcal{R}_{-i}^{*}$. However, since the assumption operator satisfies conjunction but not monotonicity, it holds for every $R_{-i}^{\prime}, R_{-i}^{\prime \prime} \in \mathcal{R}_{-i}^{*}$ that

$$
\mathcal{L}_{i}^{a}\left(R_{-i}^{\prime}\right) \cap \mathcal{L}_{i}^{a}\left(R_{-i}^{\prime \prime}\right) \subseteq \mathcal{L}_{i}^{a}\left(R_{-i}^{\prime} \cup R_{-i}^{\prime \prime}\right),
$$

while the inverse inclusion need not hold. In particular, $\mathcal{L}_{i}^{a}\left(R_{-i}^{\prime}\right) \cap \mathcal{L}_{i}^{a}\left(R_{-i}^{\prime \prime}\right) \neq \emptyset$ only if $C_{-i}\left(R_{-i}^{\prime}\right) \subseteq C_{-i}\left(R_{-i}^{\prime \prime}\right)$ or $C_{-i}\left(R_{-i}^{\prime \prime}\right) \subseteq C_{-i}\left(R_{-i}^{\prime}\right)$. Finally, Definition 5 implies

$$
\mathcal{L}_{i}^{r}\left(R_{-i}^{\prime}\right) \cap \mathcal{L}_{i}^{r}\left(R_{-i}^{\prime \prime}\right)=\mathcal{L}_{i}^{r}\left(R_{-i}^{\prime} \cup R_{-i}^{\prime \prime}\right)
$$

for every $R_{-i}^{\prime}, R_{-i}^{\prime \prime} \in \mathcal{R}_{-i}^{*}$. In particular, $\mathcal{L}_{i}^{b}\left(R_{-i}^{\prime}\right) \supseteq \mathcal{L}_{i}^{b}\left(R_{-i}^{\prime \prime}\right)$ and $\mathcal{L}_{i}^{r}\left(R_{-i}^{\prime}\right) \supseteq \mathcal{L}_{i}^{r}\left(R_{-i}^{\prime \prime}\right)$ whenever $R_{-i}^{\prime} \subseteq R_{-i}^{\prime \prime}$. This conclusion need not hold for $\mathcal{L}_{i}^{a}(\cdot)$ since a likelihood ordering $L_{i}$ may assume $A_{-i}^{\prime}$ but not $A_{-i}^{\prime \prime}$ even though $A_{-i}^{\prime} \subset A_{-i}^{\prime \prime}$. Hence, we may have $\mathcal{L}_{i}^{a}\left(R_{-i}^{\prime}\right) \nsubseteq \mathcal{L}_{i}^{a}\left(R_{-i}^{\prime \prime}\right)$ and $\mathcal{L}_{i}^{a}\left(R_{-i}^{\prime}\right) \nsupseteq \mathcal{L}_{i}^{a}\left(R_{-i}^{\prime \prime}\right)$ even though $R_{-i}^{\prime} \subset R_{-i}^{\prime \prime}$.

\section{Algorithms}

In this section we provide comparable algorithms for permissibility (the DekelFudenberg procedure), iterated admissibility, proper rationalizability, and full permissibility. To define these concepts, we need to introduce the following operators:

$$
\begin{aligned}
& a_{i}\left(S_{-i}^{\prime}\right):=\left\{s_{i} \in S_{i} \mid s_{i} \text { is not weakly dominated by any } \mu_{i} \in \Delta\left(S_{i}\right) \text { on } S_{-i}^{\prime}\right\}, \\
& b_{i}\left(S_{-i}^{\prime}\right):=\left\{s_{i} \in S_{i} \mid s_{i} \text { is not strictly dominated by any } \mu_{i} \in \Delta\left(S_{i}\right) \text { on } S_{-i}^{\prime}\right\},
\end{aligned}
$$

where $S_{-i}^{\prime}$ is a non-empty subset of $S_{-i}$. Note that $\emptyset \neq a_{i}\left(S_{-i}^{\prime}\right) \subseteq b_{i}\left(S_{-i}^{\prime}\right) \subseteq S_{i}$ for any non-empty subset $S_{-i}^{\prime}$ of $S_{-i}$.

\subsection{An algorithm for permissibility}

We first consider the Dekel-Fudenberg procedure (Dekel and Fudenberg, 1990), which is the procedure where one round of maximal elimination of weakly dominated strategies is followed by iterated maximal elimination of strictly dominated strategies. Following Brandenburger (1992), strategies surviving the Dekel-Fudenberg procedure are referred to as permissible. The formal definition is as follows.

Definition 6 (Permissibility) Consider the sequence defined by, for all players $i$, $S_{i}^{0}=S_{i}$ and, for every $n \geq 1, S_{i}^{n}=b_{i}\left(S_{-i}^{n-1}\right) \cap a_{i}\left(S_{-i}\right)$. A strategy $s_{i}$ for player $i$ is permissible if $s_{i} \in \bigcap_{n=1}^{\infty} S_{i}^{n}$. 
Since $a_{i}\left(S_{-i}\right) \subseteq b_{i}\left(S_{-i}\right)$ this corresponds to the Dekel-Fudenberg procedure: Elimination of weakly dominated strategies in the first round, followed by elimination of strictly dominated strategies in later rounds.

For our algorithmic characterization of permissibility in Proposition 1 below, the following observation is useful.

Lemma 6 Let $s_{i} \in S_{i}, A_{i} \subseteq S_{i}$ and $S_{-i}^{\prime} \subseteq S_{-i}$. Then, $s_{i}$ is strictly dominated by some $\mu_{i} \in \Delta\left(A_{i}\right)$ on $S_{-i}^{\prime}$ if and only for every $(\emptyset \neq) S_{-i}^{\prime \prime} \subseteq S_{-i}^{\prime}$ strategy $s_{i}$ is weakly dominated by some $\mu_{i}^{\prime} \in \Delta\left(A_{i}\right)$ on $S_{-i}^{\prime \prime}$.

By Lemma 6 it follows that the operator $b_{i}\left(S_{-i}^{\prime}\right)$ can be expressed as follows:

$$
b_{i}\left(S_{-i}^{\prime}\right)=\left\{s_{i} \in S_{i} \mid \exists(\emptyset \neq) S_{-i}^{\prime \prime} \subseteq S_{-i}^{\prime} \text { s.t. } s_{i} \in a_{i}\left(S_{-i}^{\prime \prime}\right)\right\},
$$

and the combined operator used to define permissibility (in Definition 6) becomes:

$$
\begin{array}{r}
b_{i}\left(S_{-i}^{\prime}\right) \cap a_{i}\left(S_{-i}\right)=\left\{s_{i} \in S_{i} \mid \exists(\emptyset \neq) S_{-i}^{\prime \prime} \subseteq S_{-i}^{\prime}\right. \\
\text { s.t. } \left.s_{i} \in a_{i}\left(S_{-i}^{\prime \prime}\right) \cap a_{i}\left(S_{-i}\right)\right\} .
\end{array}
$$

Hence, a strategy $s_{i}$ for player $i$ survives another round of the Dekel-Fudenberg procedure if there exists a subset $S_{-i}^{\prime \prime}$ of non-eliminated opponent strategy profiles such that $s_{i}$ is not weakly dominated on either $S_{-i}^{\prime \prime}$ or the set $S_{-i}$ of all opponent strategy profiles.

Consider the following algorithm, which iteratedly decreases the set of likelihood orderings for all players:

Ini For all players $i$, let $\mathcal{L}_{i}^{0}=\mathcal{L}_{i}^{*}$.

Per For every $n \geq 1$ and all players $i$, let $\mathcal{L}_{i}^{n}=\mathcal{L}_{i}^{b}\left(R_{-i}\left(\mathcal{L}_{-i}^{n-1}\right)\right)$.

From the properties of $\mathcal{L}_{i}^{b}(\cdot)$ and $R_{i}(\cdot)$, it follows that Ini and Per determine, for each player, a non-increasing sequence of sets of likelihood orderings (where non-increasing are defined w.r.t. set inclusion). As a consequence, the sequence $C_{i}\left(R_{i}\left(\mathcal{L}_{i}^{n}\right)\right)$ of choice sets is non-increasing. Since the set of likelihood orderings is finite, the algorithm converges after a finite number of rounds.

For all players $i$, let $\mathcal{L}_{i}^{\infty}:=\bigcap_{n=1}^{\infty} \mathcal{L}_{i}^{n}$ be the limiting set of likelihood orderings produced by the algorithm defined by Ini and Per.

Proposition 1 Let $G$ be a finite strategic game. Then, for all players $i$, a strategy $s_{i}$ is permissible if and only if $s_{i} \in C_{i}\left(R_{i}\left(\mathcal{L}_{i}^{\infty}\right)\right)$. 
Proof. See the appendix for a proof that applies equation (1).

As we note in the proof, the same result is obtained if the algorithm is initiated with $\mathcal{L}_{i}^{0}=\tilde{\mathcal{L}}_{i}^{*}$, including only likelihood orderings that are either trivial or partition $S_{-i}$ into a non-empty proper subset and its complement. The reason is that the belief operator is concerned only with the top level element of the likelihood ordering.

\subsection{An algorithm for iterated admissibility}

Iterated admissibility is the procedure of iterated maximal elimination of weakly dominated strategies, which can formally be defined as follows.

Definition 7 (Iterated admissibility) Consider the sequence defined by, for all players $i, S_{i}^{0}=S_{i}$ and, for every $n \geq 1, S_{i}^{n}=a_{i}\left(S_{-i}^{n-1}\right) \cap S_{i}^{n-1}$. A strategy $s_{i}$ for player $i$ survives iterated admissibility if $s_{i} \in \bigcap_{n=1}^{\infty} S_{i}^{n}$.

Consider the following algorithm, which iteratedly decreases the set of likelihood orderings for all players:

Ini For all players $i$, let $\mathcal{L}_{i}^{0}=\mathcal{L}_{i}^{*}$.

IA For every $n \geq 1$ and all players $i$, let

$$
\mathcal{L}_{i}^{n}=\mathcal{L}_{i}^{a}\left(R_{-i}\left(\mathcal{L}_{-i}^{n-1}\right)\right) \cap \mathcal{L}_{i}^{n-1}
$$

It follows directly that Ini and IA determine, for each player, a non-increasing sequence of sets of likelihood orderings. As a consequence, the sequence $C_{i}\left(R_{i}\left(\mathcal{L}_{i}^{n}\right)\right)$ of choice sets is non-increasing. Since the set of likelihood orderings is finite, the algorithm converges after a finite number of rounds.

For all players $i$, let $\mathcal{L}_{i}^{\infty}:=\bigcap_{n=1}^{\infty} \mathcal{L}_{i}^{n}$ be the limiting set of likelihood orderings produced by the algorithm defined by Ini and IA.

Proposition 2 Let $G$ be a finite strategic game. Then, for all players $i$, a strategy $s_{i}$ survives iterated admissibility if and only if $s_{i} \in C_{i}\left(R_{i}\left(\mathcal{L}_{i}^{\infty}\right)\right)$.

Proof. See the appendix.

Proposition 2 is proven by showing that, for every $n \geq 0$ and all players $i$, $C_{i}\left(R_{i}\left(\mathcal{L}_{i}^{n}\right)\right)$ equals the set of player $i$ 's strategies that survives $n+1$ rounds of iterated 
admissibility. This observation echoes Brandenburger et al.'s (2008, Theorem 9.1) epistemic characterization of strategy profiles that survives a finite number of rounds of iterated admissibility (see also the observation that Stahl, 1995, makes in his theorem) by pointing out that iterated admissibility corresponds to sets of likelihood orderings where

- strategies eliminated in a later iteration are deemed infinitely more likely than strategies eliminated in an earlier iteration, and

- surviving strategies are deemed infinitely more likely than strategies eliminated in any iteration.

Thus, when evaluating iterated admissibility by considering how our algorithm eliminates likelihood orderings, our evaluation is consistent with Brandenburger et al.'s (2008, Theorem 9.1) epistemic characterization for finite numbers of iterations. ${ }^{2}$

\subsection{An algorithm for proper rationalizability}

We then consider proper rationalizability, a concept defined by Schuhmacher (1999) and characterized by Asheim (2001). We refer to these references for details.

Consider the following algorithm, which iteratedly decreases the set of likelihood orderings for all players:

Ini For all players $i$, let $\mathcal{L}_{i}^{0}=\mathcal{L}_{i}^{*}$.

PR For every $n \geq 1$ and all players $i$, let $\mathcal{L}_{i}^{n}=\mathcal{L}_{i}^{r}\left(R_{-i}\left(\mathcal{L}_{-i}^{n-1}\right)\right)$.

From the properties of $\mathcal{L}_{i}^{r}(\cdot)$ and $R_{i}(\cdot)$, it follows that Ini and PR determine, for each player, a non-increasing sequence of sets of likelihood orderings. As a consequence, the sequence $C_{i}\left(R_{i}\left(\mathcal{L}_{i}^{n}\right)\right)$ of choice sets is non-increasing. Since the set of likelihood orderings is finite, the algorithm converges after a finite number of rounds.

For all players $i$, let $\mathcal{L}_{i}^{\infty}:=\bigcap_{n=1}^{\infty} \mathcal{L}_{i}^{n}$ be the limiting set of likelihood orderings produced by the algorithm defined by $\mathbf{I n i}$ and $\mathbf{P R}$.

\footnotetext{
${ }^{2}$ Brandenburger et al. (2008) do not provide an epistemic foundation for iterated admissibility with a countably infinite number of iterations, cf. their Theorem 10.1. Barelli and Galanis (2013), Dekel et al. (2016), Keisler and Lee (2015), Lee (2016) and Yang (2015) are later papers that discuss iterated admissibility and the problem of providing an epistemic foundation for this procedure.
} 
Proposition 3 Let $G$ be a finite strategic game. Then, for all players $i$, a strategy $s_{i}$ is properly rationalizable if and only if $s_{i} \in C_{i}\left(R_{i}\left(\mathcal{L}_{i}^{\infty}\right)\right)$.

Proof. Perea (2011).

\subsection{An algorithm for full permissibility}

We finally consider an algorithm for the concept of fully permissible sets, as defined by Asheim and Dufwenberg (2003a) for 2-player games. Full permissibility selects sets of strategies, rather than individual strategies, for both players. In analogy with the combined operator for permissibility, as interpreted in (1), let a strategy subset $A_{i}$ for player $i$ survive another iteration of the procedure that defines full permissibility if there exists a union $A_{j}^{1} \cup \cdots \cup A_{j}^{n}$ of non-eliminated opponent strategy subsets $A_{j}^{1}, \ldots, A_{j}^{n}$ such that $A_{i}$ equals the set of player $i$ strategies that are not weakly dominated on the union $A_{j}^{1} \cup \cdots \cup A_{j}^{n}$ and not weakly dominated on the set $S_{j}$ of all opponent strategies.

Formally, for both players $i$, let $\Sigma_{i}$ denote the collection of all non-empty subsets of player $i$ 's strategy set $S_{i}$, and introduce the following operator:

$$
\alpha_{i}\left(\Sigma_{j}^{\prime}\right):=\left\{A_{i} \in \Sigma_{i} \mid \exists(\emptyset \neq) \Sigma_{j}^{\prime \prime} \subseteq \Sigma_{j}^{\prime} \text { s.t. } A_{i}=a_{i}\left(\cup_{A_{j} \in \Sigma_{j}^{\prime \prime}} A_{j}\right) \cap a_{i}\left(S_{j}\right)\right\},
$$

where $j \neq i$ and $\Sigma_{j}^{\prime}$ and $\Sigma_{j}^{\prime \prime}$ are non-empty subcollections of $\Sigma_{j}$. Note that $\emptyset \neq$ $\alpha_{i}\left(\Sigma_{j}^{\prime}\right) \subseteq \Sigma_{i}$ for any non-empty subcollection $\Sigma_{j}^{\prime}$ of $\Sigma_{j}$.

Definition 8 (Full permissibility) Consider the sequence defined by, for both players $i, \Sigma_{i}^{0}=\Sigma_{i}$ and, for every $n \geq 1, \Sigma_{i}^{n}=\alpha_{i}\left(\Sigma_{j}^{n-1}\right)$. A strategy set $A_{i}$ for player $i$ is fully permissible if $A_{i} \in \bigcap_{n=1}^{\infty} \Sigma_{i}^{n}$.

Consider the following algorithm, which iteratedly decreases the set of likelihood orderings for all players:

Ini For all players $i$, let $\mathcal{L}_{i}^{0}=\mathcal{L}_{i}^{*}$.

FP For every $n \geq 1$ and all players $i$, let

$$
\begin{aligned}
\mathcal{L}_{i}^{n}=\left\{L_{i} \in \mathcal{L}_{i}^{*} \mid \exists(\emptyset \neq) \mathcal{L}_{-i} \subseteq \mathcal{L}_{-i}^{n-1} \text { s.t. } L_{i}=\left(S_{-i}^{\prime}, S_{-i} \backslash S_{-i}^{\prime}\right) \text { if } S_{-i}^{\prime} \neq S_{-i}\right. \\
\left.\quad \text { and } L_{i}=\left(S_{-i}\right) \text { otherwise, where } S_{-i}^{\prime}=\cup_{L_{-i} \in \mathcal{L}_{-i}} C_{-i}\left(R_{-i}\left(L_{-i}\right)\right)\right\} .
\end{aligned}
$$


Hence, $\mathcal{L}_{i}^{n}$ contains likelihood orderings that assume a subset $S_{-i}^{\prime}$ of opponent strategy profiles (other than the set $S_{-i}$ of all opponent strategy profiles) only if $S_{-i}^{\prime}$ is a union of Cartesian products of opponent choice sets, where the union is taken over vectors of sets of opponent preference restrictions for vectors of singleton sets of likelihood orderings in some non-empty subset $\mathcal{L}_{-i}$ of $\mathcal{L}_{-i}^{n-1}$.

It follows that Ini and FP determine, for each player, a non-increasing sequence of sets of likelihood orderings. As a consequence, the sequence $\left(C_{i}\left(R_{i}\left(L_{i}\right)\right)_{L_{i} \in \mathcal{L}_{i}^{n}}\right.$ of collections of choice sets is non-increasing. Since the set of likelihood orderings is finite, the algorithm converges after a finite number of rounds.

For all players $i$, let $\mathcal{L}_{i}^{\infty}:=\bigcap_{n=1}^{\infty} \mathcal{L}_{i}^{n}$ be the limiting set of likelihood orderings produced by the algorithm defined by Ini and $\mathbf{F P}$.

Proposition 4 Let $G$ be a finite 2-player strategic game. Then, for both players $i, A_{i}$ is a fully permissible set if and only if there exists $L_{i} \in \mathcal{L}_{i}^{\infty}$ such that $A_{i}=$ $C_{i}\left(R_{i}\left(L_{i}\right)\right)$.

Proof. See the appendix.

We can use the algorithm defined by Ini and FP to define the concept of fully permissible sets for games with more than two players:

Definition 9 Let $G$ be a finite strategic game. Then, for all players $i, A_{i}$ is a fully permissible set if there exists $L_{i} \in \mathcal{L}_{i}^{\infty}$ such that $A_{i}=C_{i}\left(R_{i}\left(L_{i}\right)\right)$.

As for permissibility, we can initiate the algorithm for full permissibility with $\mathcal{L}_{i}^{0}=\tilde{\mathcal{L}}_{i}^{*}$, including only likelihood orderings that are either trivial or partition $S_{-i}$ into a non-empty proper subset and its complement. Indeed, $\mathcal{L}_{i}^{n} \subseteq \tilde{\mathcal{L}}_{i}^{*}$ for every $n \geq 1$ and all players $i$ also when the algortithm is initiated with $\mathcal{L}_{i}^{0}=\mathcal{L}_{i}^{*}$.

\section{Applying the algorithms}

In this section we put the algorithms to work. In the first subsection four examples illustrate how the algorithms lead to sequences of sets of likelihood orderings. This sheds light on differences between iterated admissibility, proper rationalizability and full permissibility. Iterated admissibility results in a strict refinement of permissibility in all four examples, proper rationalizability strictly refines permissibility in examples 2 and 3 , and full permissibility strictly refines permissibility in examples 
2 and 4. However, even when two different concepts (like iterated admissibility and proper rationalizability in examples 2 and 3) give rise to the same prescription, there are interesting differences in the working of the algorithms in terms of the likelihood orderings that are eliminated along the way. In particular, example 3 illustrates how iterated admissibility and proper rationalizability promote backward induction through two different sequences of elimination, while example 4 does the same for how iterated admissibility and full permissiblity promote forward induction.

In the second subsection we build on insights conveyed by the examples and provide through Proposition 5 a sufficient condition ensuring that any properly rationalizable strategy survives iterated admissibility. In particular, since proper equilibrium always exists and any strategy being used with positive probability in a proper equilibrium is properly rationalizable, we reach the following conclusion: If a game, for which iterated admissibility leads to a unique strategy for each player, satisfies the sufficient condition of Proposition 5, then the surviving strategies are the unique properly rationalizable strategies and the corresponding strategy profile is the unique proper equilibrium.

In the third subsection we consider a contribution on commitment bargaining (Ellingsen and Miettinen, 2008) to show the usefulness and appeal of the concept of proper rationalizability in an economically relevant situation. In particular, we use the algorithm of Section 3.3 to show how proper rationalizability yields the outcomes Ellingsen and Miettinen point to in their propositions, while other concepts do not.

\subsection{Examples}

The examples are games $G_{1}-G_{4}$, which are illustrated by Figures $1-4$. The corresponding Tables 1-4 provide the order in which likelihood orderings are eliminated by the algorithms for permissibility, iterated admissibility, proper rationalizability and full permissibility in each of these examples. ${ }^{3}$

[Table 1 about here.]

In game $G_{1}$ (discussed by Asheim and Dufwenberg, 2003a) the algorithm for permissibility rules out likelihood orderings for player 2 where $D$ is at the top level, while the algorithm for proper rationalizability in addition requires that player 2

\footnotetext{
${ }^{3}$ For permissibility and full permissibility we restrict ourselves to likelihood orderings that are either trivial or partition the opponent's strategy set into a proper subset and its complement since - as noted in the main text - this is immaterial for the outcome.
} 


$$
\begin{array}{c|c|c|}
\multicolumn{1}{c}{} & \multicolumn{1}{c}{L} & \multicolumn{1}{c}{R} \\
\cline { 2 - 3 } U & 1,1 & 1,1 \\
\hline M & 0,1 & 2,0 \\
\hline & 1,0 & 0,1 \\
\cline { 2 - 3 } & &
\end{array}
$$

Figure 1: Iterated admissibility rules out properly rationalizable strategies $\left(G_{1}\right)$.

respects the preferences of player 1 by deeming $D$ infinitely less likely than $U$ (as $U$ weakly dominates $D$ and is thus preferred by player 1 ). Since this does not imply anything about the relative likelihood of $M$ and $D$, which is what the preferences of player 2 depend on, there is no elimination of likelihood orderings for player 1 . Thus, for permissibility and proper rationalizability, the algorithm converges after one round. The algorithm for full permissibility also rules out that the top level element of a surviving likelihood ordering is a singleton set containing only $R$ or $M$. However, all three concepts eliminate only strategy $D$ in this example.

In contrast, the algorithm for iterated admissibility works by eliminating all likelihood orderings for player 2 but those that assume $\{U, M\}$, thus deeming $D$ infinitely less likely than both $U$ and $M$ in the first round. This in turn means that player 2 prefers $L$ to $R$, determining $(\{L\},\{R\})$ as the sole surviving likelihood ordering for player 1 in round 2 , and that player 1 prefers $U$ to $M$, determining $(\{U\},\{M\},\{D\})$ as the sole surviving likelihood ordering for player 2 in round 3. Thus, iterated admissibility eliminates both strategies $D$ and $M$ for player 1 and strategy $R$ for player 2 .

The key difference in game $G_{1}$ between the algorithms for iterated admissibility and the other concepts is that the algorithm for iterated admissibility insists that both $U$ and $M$ be infinitely more likely than $D$, even though only $U$ weakly dominates $D$. It follows from the structure of game $G_{1}$ that player 2 prefers $L$ to $R$ if player 2 believes that $M$ is infinitely more likely than $D$. The algorithms for the other concepts do not reach this conclusion, and thus player 2 need not prefer $L$ to $R$. Under iterated admissibility the sole surviving likelihood ordering for player 1 entails the belief that $L$ is infinitely more likely than $R$, implying that player 1 prefers $D$ to $M$. Nevertheless, the sole surviving likelihood ordering for player 2 entails the belief that $D$ is infinitely less likely than $M$.

[Table 2 about here.]

Compare game $G_{1}$ to game $G_{2}$, for which both iterated admissibility and proper rationalizability prescribe only $U$ for player 1 and only $L$ for player 2 . Also in this 


$$
\begin{array}{c|c|c|}
\multicolumn{1}{c}{} & \multicolumn{1}{c}{L} & \multicolumn{1}{c}{R} \\
\cline { 2 - 3 } U & 1,1 & 1,0 \\
M & 0,1 & 2,1 \\
\hline & 1,0 & 0,1 \\
\hline
\end{array}
$$

Figure 2: IA and proper rationalizability make same prescription $\left(G_{2}\right)$.

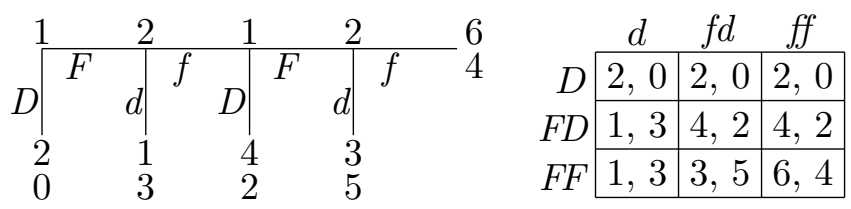

Figure 3: Backward induction in a four-legged centipede game $\left(G_{3}\right)$.

game, the algorithm for proper rationalizability rules out all likelihood orderings for player 2 but those where $D$ is infinitely less likely than $U$ (as $U$ weakly dominates $D$ and is thus preferred by player 1), while the algorithm for iterated admissibility goes further by eliminating all likelihood orderings but those where $D$ is infinitely less likely than both $U$ and $M$ in the first round. However, in this example the preferences of player 2 depends on the relative likelihood of $U$ and $D$ and thus $U$ being infinitely more likely than $D$ is sufficient for player 2 preferring $L$ to $R$. For both algorithms this determines $(\{L\},\{R\})$ as the sole surviving likelihood ordering for player 1 in round 2, and implies that player 1 prefers both $U$ and $D$ to $M$.

In the algorithm for iterated admissibility this entails that player 2 assumes $\{U\}$, implying that $U$ is infinitely more likely than both $M$ and $D$. Since all likelihood orderings but those where both $U$ and $M$ are infinitely more likely than $D$ have already been eliminated, $(\{U\},\{M\},\{D\})$ ends up as the sole surviving likelihood ordering for player 2 in round 3. However, as player 1 prefers $D$ to $M$ and the algorithm for proper rationalizability requires player 2 to respect the preferences of player 1 , this algorithm yields $(\{U\},\{D\},\{M\})$ as the sole surviving likelihood ordering for player 2 in round 3.

A key observation for game $G_{2}$ is that $U$ weakly dominates $D$, and that $L$ weakly dominates $R$ on both $\{U\}$ (which is the strategy used to eliminate $D$ in the first round of iterated admissibility) and $\{U, M\}$ (which is the set of strategies for player 1 surviving the first round of iterated admissibility). The same kind of observation can be made for the centipede game, which we turn to next.

[Table 3 about here.] 
Also in the four-legged centipede game illustrated in Figure 3 both iterated admissibility and proper rationalizability make the same prescription, namely the backward induction outcome $(D, d)$. However, as for game $G_{2}$, the algorithms in terms of likelihood orderings do not coincide. In the first round, the algorithm for proper rationalizability requires that player 1 respects the preferences of player 2 by deeming $f f$ infinitely less likely than $f d$ (as $f d$ weakly dominates $f f$ and is thus preferred by player 2). The algorithm for iterated admissibility goes further by eliminating all likelihood orderings for player 1 but those that assume $\{d, f d\}$, thus deeming $f f$ infinitely less likely than both $d$ and $f d$. Even though the set of likelihood orderings for player 1 that assume $\{d, f d\}$ is a strict subset of those that deem $f d$ infinitely more likely than $f f$, it turns out that deeming $f d$ infinitely more likely than $f f$ is sufficient for player 1 to prefer $F D$ to $F F$. Likewise, in the second round, even though the set of likelihood orderings for player 2 that assume $\{D, F D\}$ is a strict subset of those that deem $F D$ infinitely more likely than $F F$, it turns out that deeming $F D$ infinitely more likely than $F F$ is sufficient for player 2 to prefer $d$ to $f d$.

Note that in the second round, $F D$ weakly dominates $F F$ on both $\{f d\}$ (which is the strategy used to eliminate $f f$ in the first round of iterated admissibility) and $\{d, f d\}$ (which is the set of strategies for player 2 surviving the first round of iterated admissibility). Likewise, in the third round, $d$ weakly dominates $f d$ and $f f$ on both $\{F D\}$ (which is the strategy used to eliminate $F F$ in the second round of iterated admissibility) and $\{D, F D\}$ (which is the set of strategies for player 1 surviving the second round of iterated admissibility). Similar conclusions hold for any centipede game independent of size and illustrates how both iterated admissibility and proper rationalizability correspond to the procedure of backward induction in such games. ${ }^{4}$

The algorithm for permissibility works similarly in games $G_{2}$ and $G_{3}$ as in game $G_{1}$. In particular, in game $G_{2}$ it does not require player 2 to deem $U$ infinitely more likely than $D$ (even though $U$ weakly dominates $D$ and is thus preferred by player 1 ). Thus, this algorithm does not allow us to conclude that player 2 prefers $L$ to $R$, and therefore does not determine $(\{L\},\{R\})$ as the sole surviving likelihood ordering for player 1. In contrast, the algorithm for full permissibility does lead to $(\{L\},\{R\})$ as

\footnotetext{
${ }^{4}$ For finite perfect information games without relevant payoff ties, proper rationalizability leads to the unique profile of backward induction strategies (Schuhmacher, 1999; Asheim, 2001), and iterated admissibility leads to the backward induction outcome (see Battigalli, 1997, pp. 52-53, for relevant references). While the algorithms of Sections 3.2 and 3.3 correspond to the backward induction procedure in the subclass of centipede games, this does not hold for the whole class of finite perfect information games without relevant payoff ties.
} 


$$
\begin{array}{c|c|c|}
\multicolumn{1}{c}{} & \multicolumn{1}{c}{L} & \multicolumn{1}{c}{R} \\
\cline { 2 - 3 } U & 3,1 & 0,0 \\
\cline { 2 - 3 } M & 0,0 & 1,3 \\
\cline { 2 - 3 } D & 2,2 & 2,2 \\
\cline { 2 - 3 } & &
\end{array}
$$

Figure 4: Forward induction in the battle of the sexes with outside option $\left(G_{4}\right)$.

the sole surviving likelihood ordering for player 1 in game $G_{2}$. Hence, it prescribes the outcome $(U, L)$, thus coinciding with the algorithms for iterated admissibility and proper rationalizability in this respect. However, the algorithm for full permissibility does not directly conclude that $U$ infinitely more likely than $D$. Rather, as shown in Table 2, this conclusion is reached through a process that is more involved than for the algorithms for iterated admissibility and proper rationalizability.

\section{[Table 4 about here.]}

To illustrate the algorithm for full permissibility in another game where this concept has as much cutting power as iterated admissibility, but where in contrast to game $G_{2}$ it is more restrictive than proper rationalizability, we include the battle of the sexes with outside option as game $G_{4}$. In this game, both iterated admissibility and full permissibility prescribe the forward induction outcome $(U, L)$ (see Asheim and Dufwenberg, 2003b, p. 319). However, the process at which player 2 is lead to conclude that $U$ is infinitely more likely than $M$ (leading to a preference for $L$ over $R$ ) is different for the two algorithms. For iterated admissibility this follows directly from assuming $\{U, D\}$, thus deeming $M$ infinitely less likely than both $U$ and $D$, even though only $D$ weakly dominates $M$. For full permissibility the process is more involved, as illustrated in Table 4.

The examples of Figures 1-4 show that there are no logical relationships between proper rationalizability and full permissibility, while suggesting that iterated admissibility refines proper rationalizability and full permissibility, which in turn refine permissibility. Which of these relations are general properties? This is a question which we consider in the next subsection. 


\subsection{The relations between the algorithms}

The properties of $\mathcal{L}_{i}^{b}(\cdot)$ and $R_{i}(\cdot)$ combined with Lemma 5 imply both

$$
\begin{gathered}
\mathcal{L}_{i}^{a}\left(R_{-i}\left(\mathcal{L}_{-i}^{\prime}\right)\right) \subseteq \mathcal{L}_{i}^{b}\left(R_{-i}\left(\mathcal{L}_{-i}^{\prime}\right)\right), \\
\mathcal{L}_{i}^{r}\left(R_{-i}\left(\mathcal{L}_{-i}^{\prime}\right)\right) \subseteq \mathcal{L}_{i}^{b}\left(R_{-i}\left(\mathcal{L}_{-i}^{\prime}\right)\right), \\
\left\{L_{i} \in \mathcal{L}_{i}^{*} \mid \exists(\emptyset \neq) \mathcal{L}_{-i} \subseteq \mathcal{L}_{-i}^{\prime} \text { s.t. } L_{i}=\left(S_{-i}^{\prime}, S_{-i} \backslash S_{-i}^{\prime}\right) \text { if } S_{-i}^{\prime} \neq S_{-i} \subseteq \mathcal{L}_{i}^{b}\left(R_{-i}\left(\mathcal{L}_{-i}^{\prime}\right)\right)\right. \\
\text { and } L_{i}=\left(S_{-i} \text { otherwise, where } S_{-i}^{\prime}=\cup_{L_{-i} \in \mathcal{L}{ }_{i}} C_{-i}\left(R_{-i}\left(L_{-i}\right)\right)\right\}
\end{gathered}
$$

for any vector $\mathcal{L}_{-i}^{\prime}$ of non-empty sets of likelihood orderings for $i$ 's opponents, and

$$
\mathcal{L}_{i}^{b}\left(R_{-i}\left(\mathcal{L}_{-i}^{\prime}\right)\right) \subseteq \mathcal{L}_{i}^{b}\left(R_{-i}\left(\mathcal{L}_{-i}^{\prime \prime}\right)\right)
$$

if $\mathcal{L}^{\prime}{ }_{i} \subseteq \mathcal{L}_{-i}^{\prime \prime}$, signifying that $\mathcal{L}_{j}^{\prime} \subseteq \mathcal{L}_{j}^{\prime \prime}$ for all $j \neq i$. Thus, if $\mathcal{L}_{j}^{\prime} \subseteq \mathcal{L}_{j}^{\prime \prime}$ for all $j \neq i$, then the set of likelihood orderings determined for $i$ by Per on the basis of $\mathcal{L}_{-i}^{\prime \prime}$ is always a superset of those sets determined for $i$ by $\mathbf{I A}, \mathbf{P R}$ and $\mathbf{F P}$ on the basis of $\mathcal{L}_{-i}^{\prime}$. This means that Propositions $1-4$ can be used to establish the (already known) result that each of the concepts iterated admissibility, proper rationalizability and full permissibility refine the concept of permissibility. The examples illustrate that these refinements might be strict.

The other conjecture suggested by examples of Figures 1-4, namely that iterated admissibility refines proper rationalizability and full permissibility, is not true. Asheim and Dufwenberg (2003a, p. 216) show that there is no logical relationship between iterated admissibility and full permissibility: in their game $G_{4}$ (illustrated in Asheim and Dufwenberg, 2003a, Figure 4) strategy $b$ survives iterated admissibility but does not appear in any fully permissible set, while strategy $f$ appears in a fully permissible set but does not survive iterated admissibility. Likewise, our example in the introduction, illustrated in Figure 0 (see also Perea, 2012, p. 262), shows that there is no logical relationship between iterated admissibility and proper rationalizability: in the game of Figure 0 proper rationalizability uniquely selects strategy $Z$, whereas iterated admissibility uniquely selects strategy $Y$.

As we have seen in games $G_{2}$ and $G_{3}$, there are examples where proper rationalizability has at least as much cutting power as iterated admissibility. In the following proposition we generalize insights gained through these examples to provide a sufficient condition under which iterated admissibilty does not rule out properly rationalizable strategies. Hence, under these conditions, the restrictions on lexicographic beliefs that the procedure of iterated admissibility imposes along the way are convincing also given the prescriptions that this procedure ends up providing. 
Proposition 5 Consider a finite 2-player strategic game $G$ where the procedure of iterated admissibility leads to the sequence $\left\langle S_{1}^{n}, S_{2}^{n}\right\rangle_{n=0}^{\infty}$ of surviving strategy sets. Suppose that there exists a sequence $\left\langle A_{1}^{n}, A_{2}^{n}\right\rangle_{n=0}^{\infty}$ of strategy sets satisfying, for both players $i, A_{i}^{0}=S_{i}$ and for each $n \in \mathbb{N}$,

- $A_{i}^{n} \subseteq S_{i}^{n}$,

- if $S_{i}^{n} \neq S_{i}^{n-1}$, then, for every $s_{i} \in S_{i} \backslash S_{i}^{n}, s_{i}$ is weakly dominated by every $a_{i} \in A_{i}^{n}$ on either $\left(A_{j}^{n-1}\right.$ and $\left.S_{j}^{n-1}\right)$ or $S_{j}$,

- if $S_{i}^{n}=S_{i}^{n-1}$, then $A_{i}^{n}=A_{i}^{n-1}$.

Then, for both players $i$, if $s_{i}$ is properly rationalizable, then $s_{i} \in \bigcap_{n=1}^{\infty} S_{i}^{n}$.

Proof. See the appendix.

Both $G_{2}$ of Figure 2 and $G_{3}$ of Figure 3 can be used to illustrate Proposition 5. In $G_{2}$, the procedure of iterated admissibility yields the following sequence of strategy sets: $S_{1}^{1}=S_{1}^{2}=\{U, M\}$ and $S_{1}^{n}=\{U\}$ for $n \geq 3$, and $S_{2}^{1}=\{L, R\}$ and $S_{2}^{n}=\{L\}$ for $n \geq 2$. Choose $A_{1}^{n}=\{U\}$ for $n \geq 1$, and $A_{2}^{1}=\{L, R\}$ and $A_{2}^{n}=\{L\}$ for $n \geq 2$. It is straightforward to check that the conditions of Proposition 5 are satisfied; in particular, $L$ weakly dominates $R$ on both $A_{1}^{1}=\{U\}$ and $S_{1}^{1}=\{U, M\}$, and $U$ weakly dominates $M$ on $A_{2}^{2}=S_{2}^{2}=\{L\}$, and weakly dominates $D$ on $S_{2}$.

In $G_{3}$, the procedure of iterated admissibility yields the following sequence of strategy sets: $S_{1}^{1}=\{D, F D, F F\}, S_{1}^{2}=S_{1}^{3}=\{D, F D\}$ and $S_{1}^{n}=\{D\}$ for $n \geq 4$, and $S_{2}^{1}=S_{2}^{2}=\{d, f d\}$ and $S_{2}^{n}=\{d\}$ for $n \geq 3$. Choose $A_{1}^{1}=\{D, F D, F F\}$, $A_{1}^{2}=A_{1}^{3}=\{F D\}$ and $A_{1}^{n}=\{D\}$ for $n \geq 4$, and $A_{2}^{1}=A_{2}^{2}=\{f d\}$ and $A_{2}^{n}=\{d\}$ for $n \geq 3$. Again, it is straightforward to check that the conditions of Proposition 5 are satisfied; in particular, $F D$ weakly dominates $F F$ on both $A_{2}^{1}=\{f d\}$ and $S_{2}^{1}=$ $\{d, f d\}, d$ weakly dominates both $f d$ and $f f$ on both $A_{1}^{2}=\{F D\}$ and $S_{1}^{2}=\{D, F D\}$, and $D$ weakly dominates both $F D$ and $F F$ on $A_{2}^{3}=S_{2}^{3}=\{d\}$.

\subsection{Commitment bargaining}

The algorithms of Section 3 can be applied for the purpose of analyzing economically significant models, independently of whether the sufficient condition of Proposition 5 is satisfied. In particular, they can be used for comparing iterated admissibility 
to properly rationalizable strategies in specific strategic situations. In this subsection we consider a model of bilateral commitment bargaining due to Ellingsen and Miettinen (2008, Section I).

Ellingsen and Miettinen (2008) reexamine the problem of observable commitments in bargaining, first studied by Schelling (1956) and later formalized by Crawford (1982). Ellingsen and Miettinen (2008) extends Crawford's (1982) analysis by considering variants of iterated admissibility and refinements of Nash equilibrium. Here we show how some of the outcomes that Ellingsen and Miettinen (2008) suggest, in particular through their Lemma 2 and Proposition 2, can be obtained by using proper rationalizability instead of iterated admissibility. There is actually a mistake in their Lemma 2, but we will come back to this later.

In order to turn their strategic situation where two players bargain over real numbered fractions of a surplus of size 1 into a finite one-stage game with simultaneous moves, we introduce a smallest money unit $g$. We measure all variables in terms of numbers of the smallest money unit, and assume that $k$ units of the smallest money unit equals the total surplus (i.e., $k \cdot g=1$ ). Hence, players 1 and 2 bargain over a surplus of size $k$.

Each player $i$ chooses, simultaneously with the other, either to commit to some demand $s_{i} \in\{0,1, \ldots, k\}$ or to wait and remain uncommitted. Let $w$ denote the waiting strategy. Hence the strategy set of each player $i$ is $S_{i}=\{0,1, \ldots, k\} \cup\{w\}$. If both players choose $w$, then each player $i$ receives $\beta_{i}>1$, where $\beta_{1}+\beta_{2}=k$.

In the case with certain commitments and no commitment costs (Ellingsen and Miettinen, 2008, Section I) the payoffs are as follows: If only one player $i$ makes a commitment $s_{i}$, then $i$ receives $s_{i}$ and the other player receives $k-s_{i}$. If both players make commitments, then each player $i$ receives $x_{i}\left(s_{i}, s_{j}\right) \in\left\{s_{i}, s_{i}+1, \ldots, k-s_{j}\right\}$, with $x_{1}\left(s_{1}, s_{2}\right)+x_{2}\left(s_{2}, s_{1}\right) \leq k$, if $s_{1}+s_{2} \leq k$ and nothing otherwise.

The payoff function $u_{i}\left(s_{i}, s_{j}\right)$ of each player $i$ can be summarized as follows:

$$
u_{i}\left(s_{i}, s_{j}\right)= \begin{cases}x_{i}\left(s_{i}, s_{j}\right) & \text { if } s_{i}+s_{j} \leq k, \\ 0 & \text { if } s_{i}+s_{j}>k, \\ s_{i} & \text { if } s_{i} \neq w \text { and } s_{j}=w, \\ k-s_{j} & \text { if } s_{i}=w \text { and } s_{j} \neq w, \\ \beta_{i} & \text { if } s_{i}=w=s_{j} .\end{cases}
$$

Ellingsen and Miettinen (2008) show through the proof of their Lemma 2 that, for each player $i$, iterated admissibility leads to the elimination of $0,1, \ldots, \beta_{i}$ in the 
first round, and $\beta_{i}+1, \beta_{i}+2, \ldots, k-1$ in the second round, leaving $k$ and $w$ as the surviving strategies. Actually, with only $k$ and $w$ as the surviving strategies, $w$ is eliminated in the third round, since choosing $k$ yields player $i$ a payoff of 0 if the opponent also chooses $k$ and $k$ if the opponent chooses $w$, while choosing $w$ yields player $i$ a payoff of 0 if the opponent chooses $k$ and $\beta_{i}(<k)$ if the opponent also chooses $w$. Hence, the correct statement of Ellingsen and Miettinen's (2008) Lemma 2 is that only $k$ is iteratedly weakly undominated.

Ellingsen and Miettinen (2008) use Lemma 2 in their subsequent Proposition 2 to focus on Nash equilibria involving only the strategies $k$ and $w$ (including asymmetric equilibria where one commits to the entire surplus and the other waits), as opposed to the plethora of unrefined Nash equilibria that this game gives rise to (cf. Crawford, 1982). Their Proposition 2 states that only the two asymmetric equilibria along with the symmetric equilibrium where both claim the entire surplus are consistent with two rounds of elimination of weakly dominated strategies. This statement is correct, but it begs the question: why stop with two rounds of weak elimination? As the following proposition shows, proper rationalizability provides a reason for considering only the strategies $k$ and $w$.

Proposition 6 Consider the finite version of Ellingsen and Miettinen's (2008, Section I) bilateral commitment bargaining game with zero commitment cost. The properly rationalizable strategies for each player are to commit to the whole surplus, i.e., to choose the strategy $k$, or to wait, i.e., to choose the strategy $w$.

Proof. See the appendix.

The proof of Proposition 6 consists of two parts. The one part uses the algorithm of Section 3.3 to show that no strategy but $k$ and $w$ can be properly rationalizable. Since $w$ weakly dominates $0,1, \ldots, \beta_{j}$ for player $j$, respect of $j$ 's preferences forces player $i$ to deem $w$ infinitely more likely than each of $0,1, \ldots, \beta_{j}$. This in turn implies that $k$ weakly dominates $\beta_{i}+1, \beta_{i}+2, \ldots, k-1$ for player $i$. Hence, only $k$ and $w$ can be best responses when players are cautious.

The other part uses the result of Asheim (2001, Proposition 2) - that any strategy being used with positive probability in a proper equilibrium is properly rationalizable - to show that $k$ and $w$ are properly rationalizable. In particular, the asymmetric equilibria where one player commits to the entire surplus and the other waits are proper. In addition, there is a proper equilibrium where both players 
choose $k$ with probability $1 .^{5}$ In any proper equilibrium, at most one player attains positive payoff and no strategy but $k$ and $w$ is assigned positive probability. Thus, the concept of proper equilibrium focuses precisely on the equilibria highlighted in Ellingsen and Miettinen's (2008) Proposition 2. ${ }^{6}$

Ellingsen and Miettinen (2008, Section II) also consider a variant of Crawford's (1982) bilateral commitment bargaining game where commitments are uncertain. In their Proposition 4 they show that only $k$ survives iterated admissibility if commitments are uncertain. Actually, the iterations involve one round of weak elimination, followed by two rounds of strict elimination. Hence, only $k$ is permissible, and it follows from the algorithms of Sections 3.1 and 3.3 that only $k$ is properly rationalizable (and thus, $(k, k)$ is the only proper equilibrium). In their Propositions 1 and 3 they consider costly commitments. In this case, it can be shown that every strategy surviving iterated elimination of strictly dominated strategies is properly rationalizable. Hence, in all variants considered by Ellingsen and Miettinen (2008), proper rationalizability and proper equilibrium yield the outcomes they point to in their propositions, while other concepts do not.

\section{Concluding remarks}

In our opinion, proper rationalizability is an attractive concept which is based on appealing epistemic conditions. However, its applicability has been hampered by the lack of an algorithm leading directly to the properly rationalizable strategies. With Perea's (2011) algorithm, this roadblock has been removed. Here we have compared proper rationalizability to permissibility (i.e., the Dekel-Fudenberg procedure), iterated admissibility and full permissibility by presenting comparable algorithms for the three latter concepts. Through a bilateral commitment bargaining game due to Crawford (1982) and Ellingsen and Miettinen (2008) we have illustrated the usefulness of proper rationalizability in economic applications.

\footnotetext{
${ }^{5}$ This equilibrium involves likelihood orderings where $k-1$ and $w$ are at the second level. See the Claim of the Appendix.

${ }^{6}$ Even though at most one player attains positive payoff in any perfect equilibrium, there exists, for each player $i$ and any strategy $\ell \in\left\{\beta_{i}+1, \beta_{i}+2, \ldots, k-1\right\}$, a perfect equilibrium in which player $i$ assigns positive probability to $\ell$. This requires that this player also assigns sufficient positive probability to $w$, so that $k$ is the unique best response for the other player. See the Claim of the Appendix. Hence, the concept of perfect equilibrium can not be used to rule out all equilibria but the ones highlighted in Ellingsen and Miettinen's (2008) Proposition 2.
} 
The four algorithms eliminate likelihood orderings. Likelihood orderings model cautious behavior, as they require that each player takes into all opponents strategies, also those that seem unlikely to be chosen. There might also be other interesting elimination procedures that can be captured in terms of likelihood orderings. A particularly interesting example is the reasoning-based expected utility procedure defined by Cubitt and Sugden (2011). This procedure determines, for each player and every iteration, a positive and a negative subset of the player's strategy set (the two subsets having a non-empty intersection) as follows:

(i) A set of allowable probability distribution is determined by assigning positive weight to every strategy in the opponent's positive set and zero weight to every strategy in the opponent's negative set.

(ii) The player's positive set consists of strategies being a best reply to every allowable probability distribution, while the player's negative set consists of strategies not being a best reply to any allowable probability distribution.

In terms of likelihood orderings this requires the top level element to include every strategy in the opponent's positive set and to exclude every strategy in the opponent's negative set. However, the resulting algorithm is different since the partitional nature of likelihood orderings induces cautious behavior: all opponent strategies, also those in the negative set are taken into account.

Finally, it is of interest to speculate how epistemic foundations for the algorithms of the present paper might be provided in models of interactive beliefs. Epistemic models of games of complete information usually contain a set $T_{i}$ of epistemic types for all players $i$, where every type $t_{i}$ of player $i$ determines a strategy choice $s_{i}$ and a belief on the set $T_{-i}$ of opponent type profiles.

The algorithms of the present paper indicate that the reasoning process might alternatively be captured by an epistemic model where every type $t_{i}$ of player $i$, in addition to having a belief over the set $T_{-i}$ of opponent type profiles, determines of non-empty subset $\mathcal{L}_{i}$ of likelihood orderings. As we have explored through the analysis of this paper, such subsets of likelihood orderings are sufficient to describe player $i$ 's (possibly incomplete) preferences over his own strategies in any stage of the reasoning processes. Furthermore, the collection of non-empty subsets of likelihood orderings is finite if the underlying game is finite.

However, such epistemic analysis is beyond the scope of the present paper and will be investigated in future work. 


\section{References}

Asheim, G.B. (2001). 'Proper rationalizability in lexicographic beliefs.' International Journal of Game Theory 30, 453-478.

Asheim, G.B., Dufwenberg, M., 2003a. 'Admissibility and common belief.' Games and Economic Behavavior 42, 208-234.

Asheim, G.B., Dufwenberg, M., 2003b. 'Deductive reasoning in extensive games.' Economic Journal 113, 305-325.

Asheim, G.B., Søvik, Y., 2005. 'Preference-based belief operators.' Mathematical Social Sciences 50, 61-82.

Barelli, P., Galanis, S., 2013. 'Admissibility and event-rationality.' Games and Economic Behavior 77, 21-40.

Battigalli, P. (1997). 'On rationalizability in extensive games.' Journal of Economic Theory 74, 40-61.

Blume, L., Brandenburger, A., and Dekel, E. (1991a). 'Lexicographic probabilities and choice under uncertainty.' Econometrica 59, 61-79.

Blume, L., Brandenburger, A., and Dekel, E. (1991b). 'Lexicographic probabilities and equilibrium refinements.' Econometrica 59, 81-98.

Börgers, T. (1994). 'Weak dominance and approximate common knowledge.' Journal of Economic Theory 64, 265-276.

Brandenburger, A. (1992). 'Lexicographic probabilities and iterated admissibility.' In Economic Analysis of Markets and Games (eds. P. Dasgupta, D. Gale, O. Hart, E. Maskin), pp. 282-290. Cambridge, MA: MIT Press.

Brandenburger, A., Friedenberg, A., and Keisler, H.J. (2008). 'Admissibility in games.' Econometrica 76, 307-352.

Crawford, Vincent P. (1982). 'A theory of disagreement in bargaining.' Econometrica 50, $607-37$.

Cubitt, R.P., Sugden, R., 2011. 'The reasoning-based expected utility procedure.' Games and Economic Behavavior 71, 328-338.

Dekel, E., Friedenberg, A., and Siniscalchi, M. (2016). 'Lexicographic beliefs and assumption.' Journal of Economic Theory 163, 955-985.

Dekel, E. and Fudenberg, D. (1990). 'Rational behavior with payoff uncertainty.' Journal of Economic Theory 52, 243-67. 
Ellingsen, T. and Miettinen, T. (2008). 'Commitment and conflict in bilateral bargaining.' American Economic Review 98, 1629-1635.

Lee, B.S. (2016), 'Admissibility and assumption,' Journal of Economic Theory 163, 42-72.

Keisler, H.J. and Lee, B.S. (2016), 'Common assumption of rationality,' mimeo, University of Wisconsin-Madison and Rotman School of Management.

Pearce, D.G. (1984), 'Rationalizable strategic behavior and the problem of perfection,' Econometrica 52, 1029-1050.

Perea, A. (2011). 'An algorithm for proper rationalizability.' Games and Economic Behavior $\mathbf{7 2}, 510-525$.

Perea, A. (2012). Epistemic Game Theory: Reasoning and Choice. Cambridge University Press, Cambridge, UK.

Samuelson, L. (1992). 'Dominated strategies and common knowledge.' Games and Economic Behavior 4, 284-313.

Schelling, T.C. (1956). 'An essay on bargaining.' American Economic Review 46, 281-306.

Schuhmacher, F. (1999). 'Proper rationalizability and backward induction.' International Journal of Game Theory 28, 599-615.

Stahl, D. (1995). 'Lexicographic rationality, common knowledge, and iterated admissibility.' Economics Letters 47, 155-159.

Yang, C.-C. (2015). 'Weak assumption and iterative admissibility.' Journal of Economic Theory 158, 87-101.

\section{A Proofs}

Proof of Lemma 1. Let $\mathcal{L}_{i}$ be player $i$ 's non-empty set of likelihood orderings.

Reflexivity of $\sim_{i}^{\mathcal{L}_{i}}$ and $\succsim_{i}^{\mathcal{L}_{i}}$. Consider any $\mu_{i} \in \Delta\left(S_{i}\right)$. Then, trivially, $u_{i}\left(\mu_{i}, s_{-i}\right)$ $=u_{i}\left(\mu_{i}, s_{-i}\right)$ for every $s_{-i}$, so that $\mu_{i} \sim_{i}^{\mathcal{L}_{i}} \mu_{i}$, and thus, $\mu_{i} \succsim_{i}^{\mathcal{L}_{i}} \mu_{i}$.

Irreflexivity of $\succ_{i}^{\mathcal{L}_{i}}$. Consider any $\mu_{i} \in \Delta\left(S_{i}\right)$. Then, trivially, there exists no subset of opponent strategy profiles $S_{-i}^{\prime} \subseteq S_{-i}$ such that $\mu$ weakly dominates $\mu$ on $S_{-i}^{\prime}$. Hence, $\mu_{i} \nsucc_{i}^{\mathcal{L}_{i}} \mu_{i}$.

Transitivity of $\sim_{i}^{\mathcal{L}_{i}}$. If $\mu_{i} \sim_{i}^{\mathcal{L}_{i}} \mu_{i}^{\prime}$ and $\mu_{i}^{\prime} \sim_{i}^{\mathcal{L}_{i}} \mu_{i}^{\prime \prime}$, then $u_{i}\left(\mu_{i}, s_{-i}\right)=u_{i}\left(\mu_{i}^{\prime}, s_{-i}\right)=$ $u_{i}\left(\mu_{i}^{\prime \prime}, s_{-i}\right)$ for every $s_{-i}$, so that $\mu_{i} \sim_{i}^{\mathcal{L}_{i}} \mu_{i}^{\prime \prime}$.

Transitivity of $\succ_{i}^{\mathcal{L}_{i}}$. If $\mu_{i} \succ_{i}^{\mathcal{L}_{i}} \mu_{i}^{\prime}$ and $\mu_{i}^{\prime} \succ_{i}^{\mathcal{L}_{i}} \mu_{i}^{\prime \prime}$, then, for all $L_{i}=\left(L_{i}^{1}, L_{i}^{2}, \ldots, L_{i}^{K}\right) \in \mathcal{L}_{i}$, there exists $k^{\prime} \in\{1, \ldots, K\}$ such that $\mu_{i}$ weakly dominates $\mu_{i}^{\prime}$ on $L_{i}^{1} \cup \cdots \cup L_{i}^{k^{\prime}}$ and $k^{\prime \prime} \in$ 
$\{1, \ldots, K\}$ such that $\mu_{i}^{\prime}$ weakly dominates $\mu_{i}^{\prime \prime}$ on $L_{i}^{1} \cup \cdots \cup L_{i}^{k^{\prime \prime}}$. For each $L_{i} \in \mathcal{L}_{i}$, choose $k=\min \left\{k^{\prime}, k^{\prime \prime}\right\}$. Then $\mu_{i}$ weakly dominates $\mu_{i}^{\prime \prime}$ on $L_{i}^{1} \cup \cdots \cup L_{i}^{k}$ since $u_{i}\left(\mu_{i}, s_{-i}\right) \geq u_{i}\left(\mu_{i}^{\prime}, s_{-i}\right)$ and $u_{i}\left(\mu_{i}^{\prime}, s_{-i}\right) \geq u_{i}\left(\mu_{i}^{\prime \prime}, s_{-i}\right)$ for every $s_{-i} \in L_{i}^{1} \cup \cdots \cup L_{i}^{k}$, with $u_{i}\left(\mu_{i}, s_{-i}^{\prime}\right)>u_{i}\left(\mu_{i}^{\prime}, s_{-i}^{\prime}\right)$ or $u_{i}\left(\mu_{i}^{\prime}, s_{-i}^{\prime}\right)>u_{i}\left(\mu_{i}^{\prime \prime}, s_{-i}^{\prime}\right)$ for some $s_{-i}^{\prime} \in L_{i}^{1} \cup \cdots \cup L_{i}^{k}$. Hence, for all $L_{i} \in \mathcal{L}_{i}$, there exists $k \in\{1, \ldots, K\}$ such that $\mu_{i}$ weakly dominates $\mu_{i}^{\prime \prime}$ on $L_{i}^{1} \cup \cdots \cup L_{i}^{k}$, so that so that $\mu_{i} \succ_{i}^{\mathcal{L}_{i}} \mu_{i}^{\prime \prime}$.

Transitivity of $\succsim_{i}^{\mathcal{L}_{i}}$. Consider any $\mu_{i}, \mu_{i}^{\prime}, \mu_{i}^{\prime \prime} \in \Delta\left(S_{i}\right)$ such that $\mu_{i} \succsim_{i}^{\mathcal{L}_{i}} \mu_{i}^{\prime}$ and $\mu_{i}^{\prime} \succsim_{i}^{\mathcal{L}_{i}} \mu_{i}^{\prime \prime}$. We must show that $\mu_{i} \succsim_{i}^{\mathcal{L}_{i}} \mu_{i}^{\prime \prime}$.

Case 1: If $\mu_{i} \sim_{i}^{\mathcal{L}_{i}} \mu_{i}^{\prime}$ and $\mu_{i}^{\prime} \sim_{i}^{\mathcal{L}_{i}} \mu_{i}^{\prime \prime}$, then it follows from the transitivity of $\sim_{i}^{\mathcal{L}_{i}}$ that $\mu_{i} \sim_{i}^{\mathcal{L}_{i}} \mu_{i}^{\prime \prime}$, and thus, $\mu_{i} \succsim_{i}^{\mathcal{L}_{i}} \mu_{i}^{\prime \prime}$.

Case 2a: If $\mu_{i} \succ_{i}^{\mathcal{L}_{i}} \mu_{i}^{\prime}$ and $\mu_{i}^{\prime} \sim_{i}^{\mathcal{L}_{i}} \mu_{i}^{\prime \prime}$, then since $u_{i}\left(\mu_{i}^{\prime}, s_{-i}\right)=u_{i}\left(\mu_{i}^{\prime \prime}, s_{-i}\right)$ for every $s_{-i}$, for all $L_{i}=\left(L_{i}^{1}, L_{i}^{2}, \ldots, L_{i}^{K}\right) \in \mathcal{L}_{i}$, there exists $k \in\{1, \ldots, K\}$ such that $\mu_{i}$ weakly dominates $\mu_{i}^{\prime \prime}$ on $L_{i}^{1} \cup \cdots \cup L_{i}^{k}$, so that $\mu_{i} \succ_{i}^{\mathcal{L}_{i}} \mu_{i}^{\prime \prime}$, and thus, $\mu_{i} \succsim_{i}^{\mathcal{L}_{i}} \mu_{i}^{\prime \prime}$.

Case 2b: Likewise if $\mu_{i} \sim_{i}^{\mathcal{L}_{i}} \mu_{i}^{\prime}$ and $\mu_{i}^{\prime} \succ_{i}^{\mathcal{L}_{i}} \mu_{i}^{\prime \prime}$.

Case 3: If $\mu_{i} \succ_{i}^{\mathcal{L}_{i}} \mu_{i}^{\prime}$ and $\mu_{i}^{\prime} \succ_{i}^{\mathcal{L}_{i}} \mu_{i}^{\prime \prime}$, then it follows from the transitivity of $\succ_{i}^{\mathcal{L}_{i}}$ that $\mu_{i} \succ_{i}^{\mathcal{L}_{i}} \mu_{i}^{\prime \prime}$, and thus, $\mu_{i} \succsim_{i}^{\mathcal{L}_{i}} \mu_{i}^{\prime \prime}$.

Objective independence of $\sim_{i}^{\mathcal{L}_{i}}$ Consider any $\mu_{i}, \mu_{i}^{\prime}, \mu_{i}^{\prime \prime} \in \Delta\left(S_{i}\right)$ and all $\gamma \in(0,1)$. Objective independence follows since $u_{i}\left(\mu_{i}^{\prime}, s_{-i}\right)=u_{i}\left(\mu_{i}^{\prime \prime}, s_{-i}\right)$ for every $s_{-i} \in S_{-i}$ if and only if $u_{i}\left(\gamma \mu_{i}^{\prime}+(1-\gamma) \mu_{i}^{\prime \prime \prime}, s_{-i}\right)=u_{i}\left(\gamma \mu_{i}^{\prime \prime}+(1-\gamma) \mu_{i}^{\prime \prime \prime}, s_{-i}\right)$ for every $s_{-i} \in S_{-i}$

Objective independence of $\succ_{i}^{\mathcal{L}_{i}}$. Consider any $\mu_{i}, \mu_{i}^{\prime}, \mu_{i}^{\prime \prime} \in \Delta\left(S_{i}\right)$ and all $\gamma \in(0,1)$. Objective independence follows since, for any $S_{-i}^{\prime} \subseteq S_{-i}, \mu_{i}^{\prime}$ weakly dominates $\mu_{i}^{\prime \prime}$ on $S_{-i}^{\prime}$ if and only if $\gamma \mu_{i}^{\prime}+(1-\gamma) \mu_{i}^{\prime \prime \prime}$ weakly dominates $\gamma \mu_{i}^{\prime \prime}+(1-\gamma) \mu_{i}^{\prime \prime \prime}$ on $S_{-i}^{\prime}$.

Proof of Lemma 2. Let $\mathcal{L}_{i}$ be player $i$ 's non-empty set of likelihood orderings, and consider two mixed strategies $\mu_{i}^{\prime}, \mu_{i}^{\prime \prime} \in \Delta\left(S_{i}\right)$.

Assume $\mu_{i}^{\prime} \succ_{i}^{\mathcal{L}_{i}} \mu_{i}^{\prime \prime}$. Hence, for all $L_{i}=\left(L_{i}^{1}, L_{i}^{2}, \ldots, L_{i}^{K}\right) \in \mathcal{L}_{i}$, there exists $k \in$ $\{1, \ldots, K\}$ such that $\mu_{i}^{\prime}$ weakly dominates $\mu_{i}^{\prime \prime}$ on $L_{i}^{1} \cup \cdots \cup L_{i}^{k}$. Fix $L_{i} \in \mathcal{L}_{i}$. We need to show that every LPS $\lambda_{i}$ consistent with $L_{i}$ ranks $\mu_{i}^{\prime}$ above $\mu_{i}^{\prime \prime}$. This follows since $u_{i}\left(\mu_{i}^{\prime}, \lambda_{i}^{\ell}\right) \geq$ $u_{i}\left(\mu_{i}^{\prime \prime}, \lambda_{i}^{\ell}\right)$ for all $\ell \in\{1, \ldots, k\}$ and $u_{i}\left(\mu_{i}^{\prime}, \lambda_{i}^{\ell^{\prime}}\right)>u_{i}\left(\mu_{i}^{\prime \prime}, \lambda_{i}^{\ell^{\prime}}\right)$ for some $\ell^{\prime} \in\{1, \ldots, k\}$, as $\mu_{i}^{\prime}$ weakly dominates $\mu_{i}^{\prime \prime}$ on $L_{i}^{1} \cup \cdots \cup L_{i}^{k}=\operatorname{supp} \lambda_{1} \cup \cdots \cup \operatorname{supp} \lambda_{k}$.

Assume that, for all $L_{i} \in \mathcal{L}_{i}$, every LPS $\lambda_{i}$ consistent with $L_{i}$ ranks $\mu_{i}^{\prime}$ above $\mu_{i}^{\prime \prime}$. Fix $L_{i}=\left(L_{i}^{1}, L_{i}^{2}, \ldots, L_{i}^{K}\right) \in \mathcal{L}_{i}$. We need to show that there exists $k \in\{1, \ldots, K\}$ such that $\mu_{i}^{\prime}$ weakly dominates $\mu_{i}^{\prime \prime}$ on $L_{i}^{1} \cup \cdots \cup L_{i}^{k}$. Suppose, by way of contradiction, that, for all $k \in\{1, \ldots, K\}, \mu_{i}^{\prime}$ does not weakly dominate $\mu_{i}^{\prime \prime}$ on $L_{i}^{1} \cup \cdots \cup L_{i}^{k}=\operatorname{supp} \lambda_{1} \cup \cdots \cup \operatorname{supp} \lambda_{k}$. Case 1: $u_{i}\left(\mu_{i}^{\prime}, s_{-i}\right) \leq u_{i}\left(\mu_{i}^{\prime \prime}, s_{-i}\right)$ for every $s_{-i} \in S_{-i}$. Then no LPS $\lambda_{i}$ consistent with $L_{i}$ ranks $\mu_{i}^{\prime}$ above $\mu_{i}^{\prime \prime}$. Case 2: $u_{i}\left(\mu_{i}^{\prime}, s_{-i}^{\prime}\right)>u_{i}\left(\mu_{i}^{\prime \prime}, s_{-i}^{\prime}\right)$ for some $s_{-i}^{\prime} \in S_{-i}$. W.l.o.g., choose $s_{-i}^{\prime}$ and $k \in\{1, \ldots, K\}$ with the properties that $s_{-i}^{\prime} \in L_{i}^{k}$ and $u_{i}\left(\mu_{i}^{\prime}, s_{-i}\right) \leq u_{i}\left(\mu_{i}^{\prime \prime}, s_{-i}\right)$ for every $s_{-i} \in L_{i}^{1} \cup \cdots \cup L_{i}^{k-1}$. Since $\mu_{i}^{\prime}$ does not weakly dominate $\mu_{i}^{\prime \prime}$ on $L_{i}^{1} \cup \cdots \cup L_{i}^{k}$, there exists $s_{-i}^{\prime \prime} \in L_{i}^{1} \cup \cdots \cup L_{i}^{k}=\operatorname{supp} \lambda_{1} \cup \cdots \cup \operatorname{supp} \lambda_{k}$ such that $u_{i}\left(\mu_{i}^{\prime}, s_{-i}^{\prime \prime}\right)<u_{i}\left(\mu_{i}^{\prime \prime}, s_{-i}^{\prime \prime}\right)$. Then it is possible to construct $\tilde{\lambda}_{i}$ consistent with $L_{i}$ such that $u_{i}\left(\mu_{i}^{\prime}, \tilde{\lambda}_{i}^{k}\right)<u_{i}\left(\mu_{i}^{\prime \prime}, \tilde{\lambda}_{i}^{k}\right)$ and $u_{i}\left(\mu_{i}^{\prime}, \tilde{\lambda}_{i}^{\ell}\right) \leq u_{i}\left(\mu_{i}^{\prime \prime}, \tilde{\lambda}_{i}^{\ell}\right)$ for all $\ell \in\{1, \ldots, k-1\}$, implying that $\tilde{\lambda}_{i}$ consistent with $L_{i}$ ranks $\mu_{i}^{\prime \prime}$ 
above $\mu_{i}^{\prime}$. In both cases, we obtain a contradiction to the claim that every LPS $\lambda_{i}$ consistent with $L_{i}$ ranks $\mu_{i}^{\prime}$ above $\mu_{i}^{\prime \prime}$.

Assume $\mu_{i}^{\prime} \sim_{i}^{\mathcal{L}_{i}} \mu_{i}^{\prime \prime}$. Hence, $u_{i}\left(\mu_{i}^{\prime}, s_{-i}\right)=u_{i}\left(\mu_{i}^{\prime \prime}, s_{-i}\right)$ for every $s_{-i}$. Then, clearly, every LPS $\lambda_{i}$ deem $\mu_{i}^{\prime}$ indifferent to $\mu_{i}^{\prime \prime}$.

Assume that, for all $L_{i} \in \mathcal{L}_{i}$, every LPS $\lambda_{i}$ consistent with $L_{i}$, deem $\mu_{i}^{\prime}$ indifferent to $\mu_{i}^{\prime \prime}$. We need to show that $u_{i}\left(\mu_{i}^{\prime}, s_{-i}\right)=u_{i}\left(\mu_{i}^{\prime \prime}, s_{-i}\right)$ for every $s_{-i}$. Suppose, by way of contradiction, that $u_{i}\left(\mu_{i}^{\prime}, s_{-i}^{\prime}\right) \neq u_{i}\left(\mu_{i}^{\prime \prime}, s_{-i}^{\prime}\right)$ for some $s_{-i}^{\prime} \in S_{-i}$. W.l.o.g., choose $s_{-i}^{\prime}$, $L_{i}=\left(L_{i}^{1}, L_{i}^{2}, \ldots, L_{i}^{K}\right) \in \mathcal{L}_{i}$, and $k \in\{1, \ldots, K\}$ with the properties that $s_{-i}^{\prime} \in L_{i}^{k}$ and $u_{i}\left(\mu_{i}^{\prime}, s_{-i}\right)=u_{i}\left(\mu_{i}^{\prime \prime}, s_{-i}\right)$ for every $s_{-i} \in L_{i}^{1} \cup \cdots \cup L_{i}^{k-1}$. Then it is possible to construct $\tilde{\lambda}_{i}$ consistent with $L_{i}$ such that $u_{i}\left(\mu_{i}^{\prime}, \tilde{\lambda}_{i}^{k}\right) \neq u_{i}\left(\mu_{i}^{\prime \prime}, \tilde{\lambda}_{i}^{k}\right)$ and $u_{i}\left(\mu_{i}^{\prime}, \tilde{\lambda}_{i}^{\ell}\right)=u_{i}\left(\mu_{i}^{\prime \prime}, \tilde{\lambda}_{i}^{\ell}\right)$ for all $\ell \in\{1, \ldots, k-1\}$, implying that $\tilde{\lambda}_{i}$ consistent with $L_{i}$ does not deem $\mu_{i}^{\prime}$ indifferent to $\mu_{i}^{\prime \prime}$. This contradicts that every LPS $\lambda_{i}$ consistent with $L_{i}$, deem $\mu_{i}^{\prime}$ indifferent to $\mu_{i}^{\prime \prime}$.

Proof of Remark 2. Let $\mathcal{L}_{i}$ be player $i$ 's non-empty set of likelihood orderings, and consider two pure opponent strategy profiles $s_{-i}^{\prime}, s_{-i}^{\prime \prime} \in S_{-i}$. Assume $s_{-i}^{\prime} \gg_{i}^{\mathcal{L}_{i}} s_{-i}^{\prime \prime}$. Hence, for all $L_{i}=\left(L_{i}^{1}, L_{i}^{2}, \ldots, L_{i}^{K}\right) \in \mathcal{L}_{i}$, there exists $k \in\{1, \ldots, K\}$ such that $s_{-i}^{\prime} \in$ $L_{i}^{1} \cup \cdots \cup L_{i}^{k}$ and $s_{-i}^{\prime \prime} \notin L_{i}^{1} \cup \cdots \cup L_{i}^{k}$. If the mixed strategies $\mu_{i}^{\prime}, \mu_{i}^{\prime \prime} \in \Delta\left(S_{i}\right)$ satisfy $u_{i}\left(\mu_{i}^{\prime}, s_{-i}^{\prime}\right)>u_{i}\left(\mu_{i}^{\prime \prime}, s_{-i}^{\prime}\right)$ and, for all $s_{-i} \in S_{-i} \backslash\left\{s_{-i}^{\prime}, s_{-i}^{\prime \prime}\right\}, u_{i}\left(\mu_{i}^{\prime}, s_{-i}\right)=u_{i}\left(\mu_{i}^{\prime \prime}, s_{-i}\right)$, then, for all $L_{i}=\left(L_{i}^{1}, L_{i}^{2}, \ldots, L_{i}^{K}\right) \in \mathcal{L}_{i}, \mu_{i}^{\prime}$ weakly dominates $\mu_{i}^{\prime \prime}$ on $L_{i}^{1} \cup \cdots \cup L_{i}^{k}$ by choosing $k \in\{1, \ldots, K\}$ such that $s_{-i}^{\prime} \in L_{i}^{1} \cup \cdots \cup L_{i}^{k}$ and $s_{-i}^{\prime \prime} \notin L_{i}^{1} \cup \cdots \cup L_{i}^{k}$. Thus, $\mu_{i}^{\prime} \succ_{i}^{\mathcal{L}_{i}} \mu_{i}^{\prime \prime}$ whenever the mixed strategies $\mu_{i}^{\prime}, \mu_{i}^{\prime \prime}$ have these properties.

Proof of Lemma 3. Let $\left(s_{i}, A_{i}\right) \in R_{i}\left(\mathcal{L}_{i}\right)$, implying that there exists $\mu_{i} \in \Delta\left(A_{i}\right)$ such that $\mu_{i} \succ_{i}^{\mathcal{L}_{i}} s_{i}$. Clearly, $\mu_{i}\left(s_{i}\right)<1$ since $\succ_{i}^{\mathcal{L}_{i}}$ is irreflexive (cf. Lemma 1). If $\mu_{i}\left(s_{i}\right)=0$, so that $s_{i} \notin \operatorname{supp} \mu_{i}$, then $\mu_{i} \in \Delta\left(A_{i} \backslash\left\{s_{i}\right\}\right)$ and $\mu_{i} \succ_{i}^{\mathcal{L}_{i}} s_{i}$, implying that $\left(s_{i}, A_{i}^{\prime}\right) \in R_{i}\left(\mathcal{L}_{i}\right)$ where $A_{i}^{\prime}=A_{i} \backslash\left\{s_{i}\right\}$. If $\mu_{i}\left(s_{i}\right)=(0,1)$, rewrite $\mu_{i}$ as $\mu_{i}\left(s_{i}\right) s_{i}+\left(1-\mu_{i}\left(s_{i}\right)\right) \mu_{i}^{\prime}$, where $\mu_{i}^{\prime}$ is defined by $\mu_{i}^{\prime}\left(s_{i}^{\prime}\right)=\mu_{i}\left(s_{i}^{\prime}\right) /\left(1-\mu_{i}\left(s_{i}\right)\right)$ for all $s_{i}^{\prime} \neq s_{i}$. Then

$$
\mu_{i}\left(s_{i}\right) s_{i}+\left(1-\mu_{i}\left(s_{i}\right)\right) \mu_{i}^{\prime}=\mu_{i} \succ_{i}^{\mathcal{L}_{i}} s_{i}=\mu_{i}\left(s_{i}\right) s_{i}+\left(1-\mu_{i}\left(s_{i}\right)\right) s_{i} .
$$

Hence, by the objective independence of $\succ_{i}^{\mathcal{L}_{i}}$ (cf. Lemma 1), $\mu_{i}^{\prime} \succ_{i}^{\mathcal{L}_{i}} s_{i}$, where $\mu_{i}^{\prime} \in$ $\Delta\left(A_{i} \backslash\left\{s_{i}\right\}\right)$. Thus, also in this case, $\left(s_{i}, A_{i}^{\prime}\right) \in R_{i}\left(\mathcal{L}_{i}\right)$ where $A_{i}^{\prime}=A_{i} \backslash\left\{s_{i}\right\}$.

Proof of Lemma 4. Assume there exists $\mu_{i} \in \Delta\left(S_{i}\right)$ such that $\mu_{i} \succ_{i}^{\mathcal{L}_{i}} s_{i}$. Then $\left(s_{i}, S_{i}\right) \in R_{i}\left(\mathcal{L}_{i}\right)$ and $s_{i} \in S_{i} \backslash C_{i}\left(R_{i}\left(\mathcal{L}_{i}\right)\right)$. Hence,

$$
C_{i}\left(R_{i}\left(\mathcal{L}_{i}\right)\right) \subseteq\left\{s_{i} \in S_{i} \mid \nexists \mu_{i} \in \Delta\left(S_{i}\right) \text { such that } \mu_{i} \succ_{i}^{\mathcal{L}_{i}} s_{i}\right\} .
$$

Assume there does not exist $\mu_{i} \in \Delta\left(S_{i}\right)$ such that $\mu_{i} \succ_{i}^{\mathcal{L}_{i}} s_{i}$. Then there does not exist $A_{i} \subseteq S_{i}$ with $\left(s_{i}, A_{i}\right) \in R_{i}\left(\mathcal{L}_{i}\right)$, and $s_{i} \in C_{i}\left(R_{i}\left(\mathcal{L}_{i}\right)\right)$. Hence,

$$
C_{i}\left(R_{i}\left(\mathcal{L}_{i}\right)\right) \supseteq\left\{s_{i} \in S_{i} \mid \nexists \mu_{i} \in \Delta\left(S_{i}\right) \text { such that } \mu_{i} \succ_{i}^{\mathcal{L}_{i}} s_{i}\right\} .
$$

The first part of the lemma follows from (A1) and (A2). 
To show $C_{i}\left(R_{i}\left(\mathcal{L}_{i}\right)\right) \neq \emptyset$, consider an LPS $\lambda_{i}$ that is consistent with some $L_{i} \in \mathcal{L}_{i}$. Since the ranking-above relation for given LPS $\lambda_{i}$ is transitive and irreflexive and $S_{i}$ is finite, there exists $s_{i} \in S_{i}$ such that $\lambda_{i}$ ranks no $s_{i}^{\prime} \in S_{i}$ above $s_{i}$. Moreover, by the definition of the ranking-above relation it now follows that $\lambda_{i}$ ranks no $\mu_{i} \in \Delta\left(S_{i}\right)$ above $s_{i}$. Hence, by Lemma 2 , there does not exist $\mu_{i} \in \Delta\left(S_{i}\right)$ such that $\mu_{i} \succ_{i}^{\mathcal{L}_{i}} s_{i}$, implying by the first part of the lemma that $s_{i} \in C_{i}\left(R_{i}\left(\mathcal{L}_{i}\right)\right)$.

Proof of Lemma 5. Assume that player $i$ with non-empty set $\mathcal{L}_{i}$ of likelihoods orderings respects $R_{-i}$. Suppose $s_{j}^{\prime} \notin C_{j}\left(R_{j}\right)$ for some $j \neq i$, implying that there exists $A_{j}$ such that $\left(s_{j}, A_{j}\right) \in R_{j}$. Since $i$ respects $R_{-i},\left(L_{i}^{1} \cap\left\{s_{j}\right\}\right) \times S_{-i, j}=\emptyset$ for all $L_{i}=\left(L_{i}^{1}, L_{i}^{2}, \ldots, L_{i}^{K}\right) \in \mathcal{L}_{i}$. Therefore, if $s_{-i} \in L_{i}^{1}$ for some $L_{i}=\left(L_{i}^{1}, L_{i}^{2}, \ldots, L_{i}^{K}\right) \in \mathcal{L}_{i}$, then $s_{-i} \in \prod_{j \neq i} C_{j}\left(R_{j}\right)=C_{-i}\left(R_{-i}\right)$. This implies that $L_{i} \subseteq C_{-i}\left(R_{-i}\right)$ for all $L_{i}=$ $\left(L_{i}^{1}, L_{i}^{2}, \ldots, L_{i}^{K}\right) \in \mathcal{L}_{i}$ and establishes the lemma.

Proof of Lemma 6. Only if. If there exists $\mu_{i} \in \Delta\left(A_{i}\right)$ such that $\mu_{i}$ strictly dominates $s_{i}$ on $S_{-i}^{\prime}$, then, for every $(\emptyset \neq) S_{-i}^{\prime \prime} \subseteq S_{-i}^{\prime}, \mu_{i} \in \Delta\left(A_{i}\right)$ weakly dominates $s_{i}$ on $S_{-i}^{\prime \prime}$.

If. Suppose there does not exist $\mu_{i} \in \Delta\left(A_{i}\right)$ such that $\mu_{i}$ strictly dominates $s_{i}$ on $S_{-i}^{\prime}$. Hence, by Pearce (1984, Lemma 3$)$, there exists $\lambda_{i} \in \Delta\left(S_{-i}^{\prime}\right)$ such that $u\left(s_{i}, \lambda_{i}\right) \geq u\left(s_{i}^{\prime}, \lambda_{i}\right)$ for all $s_{i}^{\prime} \in A_{i}$. Then, by Pearce (1984, Lemma 4), there does not exist $\mu_{i}^{\prime} \in \Delta\left(A_{i}\right)$ such that $\mu_{i}^{\prime}$ weakly dominates $s_{i}$ on $S_{-i}^{\prime \prime}:=\operatorname{supp} \lambda_{i} \subseteq S_{-i}^{\prime}$.

Proof of Proposition 1. Consider, for all players $i$, the sequence $\left\langle S_{i}^{n}\right\rangle_{n=0}^{\infty}$ defined in Definition 6. We show, by induction on $n$, that $C_{i}\left(R_{i}\left(\mathcal{L}_{i}^{n}\right)\right)=S_{i}^{n+1}$ for all players $i$ and every $n \geq 0$.

Part (i). For $n=0$, we have that $\mathcal{L}_{i}^{0}=\mathcal{L}_{i}^{*}$ and hence,

$R_{i}\left(\mathcal{L}_{i}^{0}\right)=\left\{\left(s_{i}, A_{i}\right) \mid \exists \mu_{i} \in \Delta\left(A_{i}\right)\right.$ such that $s_{i}$ is weakly dominated by $\mu_{i}$ on $\left.S_{-i}\right\}$.

Therefore, $C_{i}\left(R_{i}\left(\mathcal{L}_{i}^{0}\right)\right)=a_{i}\left(S_{-i}\right)=b_{i}\left(S_{-i}^{0}\right) \cap a_{i}\left(S_{-i}\right)=S_{i}^{1}$ for all players $i$, since $S_{-i}^{0}=S_{-i}$ and $a_{i}\left(S_{-i}\right) \subseteq b_{i}\left(S_{-i}\right)$.

Part (ii). Now, let $n \geq 1$, and assume that for all players $i, C_{i}\left(R_{i}\left(\mathcal{L}_{i}^{n-1}\right)\right)=S_{i}^{n}$. We show that, for all players $i, C_{i}\left(R_{i}\left(\mathcal{L}_{i}^{n}\right)\right)=S_{i}^{n+1}$.

Fix a player $i$. By definition, $\mathcal{L}_{i}^{n}=\mathcal{L}_{i}^{b}\left(R_{-i}\left(\mathcal{L}_{-i}^{n-1}\right)\right)$. We have that

$$
\begin{aligned}
\mathcal{L}_{i}^{b}\left(R_{-i}\left(\mathcal{L}_{-i}^{n-1}\right)\right) & =\left\{L_{i} \in \mathcal{L}_{i}^{*} \mid L_{i} \text { believes } C_{-i}\left(R_{-i}\left(\mathcal{L}_{-i}^{n-1}\right)\right)\right\} \\
& =\left\{L_{i} \in \mathcal{L}_{i}^{*} \mid L_{i} \text { believes } S_{-i}^{n}\right\} \\
& =\left\{L_{i} \in \mathcal{L}_{i}^{*} \mid L_{i}^{1} \subseteq S_{-i}^{n}\right\}
\end{aligned}
$$

by our induction assumption. But then,

and

$$
\begin{aligned}
R_{i}\left(\mathcal{L}_{i}^{n}\right)=\left\{\left(s_{i}, A_{i}\right) \mid\right. & \text { for every } L_{i}^{1} \subseteq S_{-i}^{n} \text { there is } \mu_{i} \in \Delta\left(A_{i}\right) \text { such that } \\
& \left.s_{i} \text { is weakly dominated by } \mu_{i} \text { on } L_{i}^{1} \text { or on } S_{-i}\right\}
\end{aligned}
$$




$$
\begin{aligned}
C_{i}\left(R_{i}\left(\mathcal{L}_{i}^{n}\right)\right) & =\left\{s_{i} \in S_{i} \mid \exists(\emptyset \neq) L_{i}^{1} \subseteq S_{-i}^{n} \text { s.t. } s_{i} \in a_{i}\left(L_{i}^{1}\right) \cap a_{i}\left(S_{-i}\right)\right\} \\
& =b_{i}\left(S_{-i}^{n}\right) \cap a_{i}\left(S_{-i}\right)=S_{i}^{n+1}
\end{aligned}
$$

by (1) and Definition 6, thus concluding the proof.

The proof above would also apply to the case where $\mathcal{L}_{i}^{0}=\tilde{\mathcal{L}}_{i}^{*}$, considering only likelihood orderings that consist of one or two levels only. The reason is that the restrictions on the sets $\mathcal{L}_{i}^{n}$ of likelihood orderings only apply to the top level of the likelihood orderings.

Proof of Proposition 2. Consider, for all players $i$, the sequence $\left\langle S_{i}^{n}\right\rangle_{n=0}^{\infty}$ defined in Definition 7. We show, by induction on $n$, that $C_{i}\left(R_{i}\left(\mathcal{L}_{i}^{n}\right)\right)=S_{i}^{n+1}$ for all players $i$ and every $n \geq 0$.

Part (i). For $n=0$, it follows by part (i) of the proof of Proposition 1, that $C_{i}\left(R_{i}\left(\mathcal{L}_{i}^{0}\right)\right)=$ $a_{i}\left(S_{-i}\right)=a_{i}\left(S_{-i}^{0}\right) \cap S_{i}=S_{i}^{1}$ for all players $i$.

Part (ii). Let $n \geq 1$, and assume that, for all players $i, C_{i}\left(R_{i}\left(\mathcal{L}_{i}^{m}\right)\right)=S_{i}^{m+1}$ for every $m \in\{0, \ldots, n-1\}$. We show that, for all players $i, C_{i}\left(R_{i}\left(\mathcal{L}_{i}^{n}\right)\right)=S_{i}^{n+1}$.

Fix a player $i$. By definition, we have that

$$
\mathcal{L}_{i}^{n}=\mathcal{L}_{i}^{a}\left(R_{-i}\left(\mathcal{L}_{-i}^{0}\right)\right) \cap \mathcal{L}_{i}^{a}\left(R_{-i}\left(\mathcal{L}_{-i}^{1}\right)\right) \cap \ldots \cap \mathcal{L}_{i}^{a}\left(R_{-i}\left(\mathcal{L}_{-i}^{n-1}\right)\right) .
$$

By the induction assumption, we know that $C_{-i}\left(R_{-i}\left(\mathcal{L}_{-i}^{m}\right)\right)=S_{i}^{m+1}$ for every $m \in\{0, \ldots, n-1\}$, and hence

$$
\begin{aligned}
\mathcal{L}_{i}^{a}\left(R_{-i}\left(\mathcal{L}_{-i}^{m}\right)\right) & =\left\{L_{i} \in \mathcal{L}_{i}^{*} \mid L_{i} \text { assumes } C_{-i}\left(R_{-i}\left(\mathcal{L}_{-i}^{m}\right)\right)\right\} \\
& =\left\{L_{i} \in \mathcal{L}_{i}^{*} \mid L_{i} \text { assumes } S_{-i}^{m+1}\right\} \\
& =\left\{L_{i} \in \mathcal{L}_{i}^{*} \mid \exists k \in\{1, \ldots, K\} \text { such that } L_{i}^{1} \cup \cdots \cup L_{i}^{k}=S_{-i}^{m+1}\right\}
\end{aligned}
$$

for every $m \in\{0, \ldots, n-1\}$. This implies that

$$
\mathcal{L}_{i}^{n}=\left\{L_{i} \in \mathcal{L}_{i}^{*} \mid \forall m \in\{1, \ldots, n\}, \exists k \in\{1, \ldots, K\} \text { such that } L_{i}^{1} \cup \cdots \cup L_{i}^{k}=S_{-i}^{m}\right\} .
$$

Therefore, $R_{i}\left(\mathcal{L}_{i}^{n}\right)$ contains exactly those preference restrictions $\left(s_{i}, A_{i}\right)$ such that $s_{i}$ is weakly dominated by some $\mu_{i} \in \Delta\left(A_{i}\right)$ on some $S_{-i}^{m}$ with $m \leq n$ :

$$
\begin{aligned}
R_{i}\left(\mathcal{L}_{i}^{n}\right)=\left\{\left(s_{i}, A_{i}\right) \mid\right. & \text { there are } m \in\{0, \ldots, n\} \text { and } \mu_{i} \in \Delta\left(A_{i}\right) \\
& \text { such that } \left.s_{i} \text { is weakly dominated by } \mu_{i} \text { on } S_{-i}^{m}\right\}
\end{aligned}
$$

and

$$
C_{i}\left(R_{i}\left(\mathcal{L}_{i}^{n}\right)\right)=a_{i}\left(S_{-i}^{0}\right) \cap a_{i}\left(S_{-i}^{1}\right) \cap \cdots \cap a_{i}\left(S_{-i}^{n}\right)=S_{i}^{n+1},
$$

which completes the proof.

Proof of Proposition 4. Consider, for both players $i$, the sequence $\left\langle\Sigma_{i}^{n}\right\rangle_{n=0}^{\infty}$ defined in Definition 8. Consider also, for both players $i$, the sequence $\left\langle\tilde{\mathcal{L}}_{i}^{n}\right\rangle_{n=0}^{\infty}$ defined by

Ini* For both players $i$, let $\tilde{\mathcal{L}}_{i}^{0}=\tilde{\mathcal{L}}_{i}^{*}$. 
and $\mathbf{F P}$. Note that $\mathcal{L}_{i}^{1} \subseteq \tilde{\mathcal{L}}_{i}^{0} \subseteq \mathcal{L}_{i}^{0}$, so by induction, for every $n \geq 1, \mathcal{L}_{i}^{n+1} \subseteq \tilde{\mathcal{L}}_{i}^{n} \subseteq \mathcal{L}_{i}^{n}$. Since also the algorithm defined by Ini* and FP converges after a finite number of rounds, as the set of likelihood orderings is finite, we have that $\tilde{\mathcal{L}}_{i}^{\infty}:=\bigcap_{n=1}^{\infty} \tilde{\mathcal{L}}_{i}^{n}$ equals $\mathcal{L}_{i}^{\infty}$. Thus, it is sufficient to show that there exists $L_{i} \in \tilde{\mathcal{L}}_{i}^{n}$ such that $A_{i}=C_{i}\left(R_{i}\left(L_{i}\right)\right)$ if and only if $A_{i} \in \Sigma_{i}^{n+1}$, for both players $i$ and every $n \geq 0$. We show this by induction on $n$.

Part (i). For $n=0$, we have that $\tilde{\mathcal{L}}_{i}^{0}=\tilde{\mathcal{L}}_{i}^{*}$ and thus, $L_{i} \in \tilde{\mathcal{L}}_{i}^{0}$ if and only if $L_{i}=\left(L_{i}^{1}\right)=S_{j}$ or $L_{i}=\left(L_{i}^{1}, L_{i}^{2}\right)=\left(S_{j}^{\prime}, S_{j} \backslash S_{j}^{\prime}\right)$ for some non-empty proper subset $S_{j}^{\prime}$ of $S_{j}$. Hence, there is $L_{i} \in \tilde{\mathcal{L}}_{i}^{0}$ such that $\left(s_{i}, A_{i}\right) \in R_{i}\left(L_{i}\right)$ if and only if there exist $(\emptyset \neq) S_{j}^{\prime} \subseteq S_{j}$ and $\mu_{i} \in \Delta\left(A_{i}\right)$ such that $s_{i}$ is weakly dominated by $\mu_{i}$ on $S_{j}^{\prime}$ or $S_{j}$. Therefore, there is $L_{i} \in \tilde{\mathcal{L}}_{i}^{0}$ such that $A_{i} \in C_{i}\left(R_{i}\left(L_{i}\right)\right)$ if and only if $A_{i}=a_{i}\left(S_{j}^{\prime}\right) \cap a_{i}\left(S_{j}\right)$ for some $(\emptyset \neq) S_{j}^{\prime} \subseteq S_{j}$. It now follows from the definition of the operator $\alpha_{i}\left(\Sigma_{j}^{\prime}\right)$ that there is $L_{i} \in \tilde{\mathcal{L}}_{i}^{0}$ such that $A_{i} \in C_{i}\left(R_{i}\left(L_{i}\right)\right)$ if and only if $A_{i} \in \alpha_{i}\left(\Sigma_{j}\right)=\alpha_{i}\left(\Sigma_{j}^{0}\right)=\Sigma_{i}^{1}$, since $\Sigma_{j}^{0}=\Sigma_{j}$.

Part (ii). Now, let $n \geq 1$, and assume that for both players $i$, there exists $L_{i} \in \tilde{\mathcal{L}}_{i}^{n-1}$ such that $A_{i}=C_{i}\left(R_{i}\left(L_{i}\right)\right)$ if and only if $A_{i} \in \Sigma_{i}^{n}$.

Fix a player $i$. By FP, $L_{i} \in \tilde{\mathcal{L}}_{i}^{n}$ is equivalent to there existing $(\emptyset \neq) \mathcal{L}_{j} \subseteq \tilde{\mathcal{L}}_{j}^{n-1}$ such that $L_{i}=\left(S_{j}^{\prime}, S_{j} \backslash S_{j}^{\prime}\right)$ if $S_{j}^{\prime} \neq S_{j}$ and $L_{i}=\left(S_{j}\right)$ otherwise, where $S_{j}^{\prime}=\cup_{L_{j} \in \mathcal{L}_{j}} C_{j}\left(R_{j}\left(L_{j}\right)\right)$. By the induction assumption this is equivalent to there existing $(\emptyset \neq) \Sigma_{j}^{\prime \prime} \subseteq \Sigma_{j}^{n}$ such that $L_{i}=\left(S_{j}^{\prime}, S_{j} \backslash S_{j}^{\prime}\right)$ if $S_{j}^{\prime} \neq S_{j}$ and $L_{i}=\left(S_{j}\right)$ otherwise, where $S_{j}^{\prime}=\cup_{A_{j} \in \Sigma_{j}^{n-1}} A_{j}$. Therefore, there is $L_{i} \in \tilde{\mathcal{L}}_{i}^{n}$ such that $A_{i}=C_{i}\left(R_{i}\left(L_{i}\right)\right)$ if and only if $A_{i}=a_{i}\left(\cup_{A_{j} \in \Sigma_{j}^{\prime \prime}} A_{j}\right) \cap a_{i}\left(S_{j}\right)$ for some $(\emptyset \neq) \Sigma_{j}^{\prime \prime} \subseteq \Sigma_{j}^{n}$. It now follows from the definition of the operator $\alpha_{i}\left(\Sigma_{j}^{\prime}\right)$ that there is $L_{i} \in \tilde{\mathcal{L}}_{i}^{n}$ such that $A_{i}=C_{i}\left(R_{i}\left(L_{i}\right)\right)$ if and only if $A_{i} \in \alpha_{i}\left(\Sigma_{j}^{n}\right)=\Sigma_{i}^{n+1}$, which completes the proof.

Proof of Proposition 5. Let $\left\langle\mathcal{L}_{1}^{n}, \mathcal{L}_{2}^{n}\right\rangle_{n=1}^{\infty}$ be the sequence of likelihood orderings according to the algorithm for proper rationalizability (cf. Section 3.3). It is sufficient to show, under the assumptions of the proposition, that for every $n \geq 0$ and both players $i$, it holds that, for every $s_{i} \in S_{i} \backslash S_{i}^{n+1},\left(s_{i},\left\{a_{i}\right\}\right) \in R_{i}\left(\mathcal{L}_{i}^{n}\right)$ for every $a_{i} \in A_{i}^{n+1}$. In this case, namely, every properly rationalizable strategy is in $\bigcap_{n=1}^{\infty} S_{i}^{n}$. We show by induction that the statement above is true.

Part (i). Let $n=0$. If $S_{i}^{1}=S_{i}$, so that there is no $s_{i} \in S_{i} \backslash S_{i}^{1}$, then the statement is trivially true. If $S_{i}^{1} \neq S_{i}$, then, by the premise of the proposition, for every $s_{i} \in S_{i} \backslash S_{i}^{1}$, $s_{i}$ is weakly dominated by every $a_{i} \in A_{i}^{1}$ on $S_{j}$. Hence, by the full support assumption, $\left(s_{i},\left\{a_{i}\right\}\right) \in R_{i}\left(\mathcal{L}_{i}^{*}\right)=R_{i}\left(\mathcal{L}_{i}^{0}\right)$, implying that the statement is true also in this case.

Part (ii). Let $n \geq 1$, and assume that, for every $m \in\{0, \ldots, n-1\}$ and both players $i$, it holds that, for every $s_{i} \in S_{i} \backslash S_{i}^{m+1},\left(s_{i},\left\{a_{i}\right\}\right) \in R_{i}\left(\mathcal{L}_{i}^{m}\right)$ for every $a_{i} \in A_{i}^{m+1}$.

Fix a player $i$. We first make the observation that, for every $m \in\{1, \ldots, n\}$, every $L_{i}=\left(L_{i}^{1}, \ldots, L_{i}^{K}\right) \in \mathcal{L}_{i}^{m}$ satisfies that there exists $k \in\{1, \ldots, K\}$ such that $A_{j}^{m} \subseteq L_{i}^{1} \cup$ $\cdots \cup L_{i}^{k} \subseteq S_{j}^{m}$. This is true by the full support assumption if $S_{j}^{m}=S_{j}$ (and thus $A_{j}^{m}=S_{j}$, by the last bullet point of Proposition 5 and fact that $A_{j}^{0}=S_{j}$ ). Assume now $S_{j}^{m} \neq S_{j}$. By the algorithm for proper rationalizability, every $L_{i} \in \mathcal{L}_{i}^{m}$ respects $R_{j}\left(\mathcal{L}_{j}^{m-1}\right)$, implying 
that there exists $k \in\{1, \ldots, K\}$ such that $\left(L_{i}^{1} \cup \cdots \cup L_{i}^{k}\right) \cap\left\{a_{j}\right\} \neq \emptyset$ for every $a_{j} \in A_{j}^{m}$ and $\left(L_{i}^{1} \cup \cdots \cup L_{i}^{k}\right) \cap\left\{s_{j}\right\}=\emptyset$ for every $s_{j} \in S_{j} \backslash S_{j}^{m}$, and the observation follows also in this case.

If $S_{i}^{n+1}=S_{i}$, then the statement is trivially true also for $n \geq 1$.

If $S_{i}^{n+1} \neq S_{i}$, let $(0 \leq) m \leq n$ satisfy $S_{i}^{n+1}=S_{i}^{m+1} \neq S_{i}^{m}$. By a premise of the proposition, for every $s_{i} \in S_{i} \backslash S_{i}^{m+1}, s_{i}$ is weakly dominated by every $a_{i} \in A_{i}^{m+1}$ on either $\left(A_{j}^{m}\right.$ and $S_{j}^{m}$ ) or $S_{j}$. If $s_{i}$ is weakly dominated by $a_{i}$ on $A_{j}^{m}$ and $S_{j}^{m}$, then $s_{i}$ is weakly dominated by $a_{i}$ on each strategy set $S_{j}^{\prime}$ satisfying $A_{j}^{m} \subseteq S_{j}^{\prime} \subseteq S_{j}^{m}$. By the observation that every $L_{i}=\left(L_{i}^{1}, \ldots, L_{i}^{K}\right) \in \mathcal{L}_{i}^{m}$ satisfies that there exists $k \in\{1, \ldots, K\}$ such that $A_{j}^{m} \subseteq L_{i}^{1} \cup \cdots \cup L_{i}^{k} \subseteq S_{j}^{m}$ it follows that $\left(s_{i},\left\{a_{i}\right\}\right) \in R_{i}\left(\mathcal{L}_{i}^{m}\right)$. If $s_{i}$ is weakly dominated by $a_{i}$ on $S_{j}$, then by the full support assumption, $\left(s_{i},\left\{a_{i}\right\}\right) \in R_{i}\left(\mathcal{L}_{i}^{*}\right)=R_{i}\left(\mathcal{L}_{i}^{0}\right)$. Hence, since the sequence of sets of likelihood orderings is non-increasing, so that $\mathcal{L}_{i}^{n} \subseteq \mathcal{L}_{i}^{m} \subseteq \mathcal{L}_{i}^{0}$ and thus, $R_{i}\left(\mathcal{L}_{i}^{n}\right) \supseteq R_{i}\left(\mathcal{L}_{i}^{m}\right) \supseteq R_{i}\left(\mathcal{L}_{i}^{0}\right)$, for every $s_{i} \in S_{i} \backslash S_{i}^{n+1},\left(s_{i},\left\{a_{i}\right\}\right) \in R_{i}\left(\mathcal{L}_{i}^{n}\right)$ for every $a_{i} \in A_{i}^{n+1}$.

Proof of Proposition 6. The proof is divided into two parts. In part (i) we show that the strategies in $S_{i} \backslash(\{k\} \cup\{w\})$ are not properly rationalizable. In part (ii) we show that $k$ and $w$ are properly rationalizable.

Part (i). Let $\left\langle\mathcal{L}_{1}^{n}, \mathcal{L}_{2}^{n}\right\rangle_{n=1}^{\infty}$ be the sequence of sets of likelihood orderings for the finite version of Ellingsen and Miettinen's (2008, Section I) bilateral commitment bargaining game with zero commitment cost, according to the algorithm for proper rationalizability (cf. Section 3.3). In order to show that the strategies in $S_{i} \backslash(\{k\} \cup\{w\})=\{0,1, \ldots, k-1\}$ are not properly rationalizable, it is sufficient to show that for each player $i$, it holds that (a) for every $s_{i} \in\left\{0,1, \ldots, \beta_{i}\right\},\left(s_{i},\{w\}\right) \in R_{i}\left(\mathcal{L}_{i}^{0}\right)$, and (b) for every $s_{i} \in\left\{\beta_{i}+1, \beta_{i}+2, \ldots, k-1\right\}$, $\left(s_{i},\{k\}\right) \in R_{i}\left(\mathcal{L}_{i}^{1}\right)$, keeping in mind that the sequence of sets of likelihood orderings is non-increasing, so that $\mathcal{L}_{i}^{n} \subseteq \mathcal{L}_{i}^{1} \subseteq \mathcal{L}_{i}^{0}$ and thus, $R_{i}\left(\mathcal{L}_{i}^{n}\right) \supseteq R_{i}\left(\mathcal{L}_{i}^{1}\right) \supseteq R_{i}\left(\mathcal{L}_{i}^{0}\right)$ for every $n \geq 1$.

Result (a) follows from the fact that, for each player $i$ and for every $s_{i} \in\left\{0,1, \ldots, \beta_{i}\right\}$, $w$ weakly dominates $s_{i}$ on $S_{j}$. (To see this, note that if the opponent chooses $w$, then player $i$ 's payoff by choosing $w$ is $\beta_{i}$, while it is $\left\{0,1, \ldots, \beta_{i}\right\}$ if player $i$ commits to one of these demands, and if the opponent chooses $s_{j} \in\{0,1, \ldots, k\}$, then player $i$ 's payoff by choosing $w$ is $1-s_{j}$, while it is no more than $1-s_{j}$ and sometimes 0 if $s_{i} \in\left\{0,1, \ldots, \beta_{i}\right\}$.) Hence, for each player $i$ and for every $s_{i} \in\left\{0,1, \ldots, \beta_{i}\right\},\left(s_{i},\{w\}\right) \in R_{i}\left(\mathcal{L}_{i}^{*}\right)=R_{i}\left(\mathcal{L}_{i}^{0}\right)$. This result implies that, for each player $i$, every $L_{i}=\left(L_{i}^{1}, \ldots, L_{i}^{K}\right) \in \mathcal{L}_{i}^{1}=\mathcal{L}_{i}^{r}\left(R_{j}\left(\mathcal{L}_{j}^{0}\right)\right)$ satisfies that there exists $k \in\{1, \ldots, K\}$ such that $\{w\} \subseteq L_{i}^{1} \cup \cdots \cup L_{i}^{k} \subseteq\left\{\beta_{j}+1, \beta_{j}+2, \ldots, k\right\} \cup\{w\}$. Result (b) follows from the fact that, for each player $i$ and for every $s_{i} \in\left\{\beta_{i}+1, \beta_{i}+2, \ldots, k-1\right\}, k$ weakly dominates $s_{i}$ on each strategy set $S_{j}^{\prime}$ satisfying $\{w\} \subseteq S_{j}^{\prime} \subseteq\left\{\beta_{j}+1, \beta_{j}+2, \ldots, k\right\} \cup\{w\}$. Hence, for each player $i$ and for every $s_{i} \in\left\{\beta_{i}+1, \beta_{i}+2, \ldots, k-1\right\},\left(s_{i},\{k\}\right) \in R_{i}\left(\mathcal{L}_{i}^{1}\right)$.

Part (ii). We establish that $k$ and $w$ are properly rationalizable in the finite version of Ellingsen and Miettinen's (2008, Section I) bilateral commitment bargaining game with zero commitment cost, by showing that both $k$ and $w$ can be used with positive probability in a proper equilibrium; thus, they are properly rationalizable (Asheim, 2001, Proposition 
2). To prove this claim, consider the likelihood orderings

$$
\begin{aligned}
& L_{1}=\left\{\{w\},\{1\},\{2\}, \ldots,\left\{\beta_{2}-1\right\},\{k\},\{k-1\}, \ldots,\left\{\beta_{2}+1\right\},\left\{\beta_{2}\right\},\{0\}\right\}, \\
& L_{2}=\left\{\{k\},\{k-1\}, \ldots,\left\{\beta_{1}+1\right\},\{w\},\left\{\beta_{1}\right\},\left\{\beta_{1}-1\right\}, \ldots,\{1\},\{0\}\right\}
\end{aligned}
$$

Since each element in either of these partitions contains only one strategy, they determine a pair of LPSs. It is straightforward to check that this pair of LPSs determines a proper equilibrium, according to Blume et al.'s (1991b, Proposition 5) characterization, where player 1 chooses $k$ with probability 1 and player 2 chooses $w$ with probability 1 .

Claim Consider the finite version of Ellingsen and Miettinen's (2008, Section I) bilateral commitment bargaining game with zero commitment cost. Assume that $x_{1}\left(s_{1}, s_{2}\right)=s_{1}$ and $x_{2}\left(s_{2}, s_{1}\right)=s_{2}$ if $s_{1}+s_{2} \leq k$.

(i) There exists a proper equilibrium where both players assign probability 1 to $k$.

(ii) For both players $i$ and any strategy $\ell \in\left\{\beta_{i}+1, \beta_{i}+2, \ldots, k-1\right\}$, there exists a perfect equilibrium where player $i$ assigns positive probability to both $w$ and $\ell$ and player $j$ assigns probability 1 to $k$.

Proof. Part (i). Consider the LPSs

$$
\begin{aligned}
& \lambda_{1}=\left\{\lambda_{1}^{1}, \ldots, \lambda_{1}^{k+1}\right\} \\
& \lambda_{2}=\left\{\lambda_{2}^{1}, \ldots, \lambda_{2}^{k+1}\right\},
\end{aligned}
$$

where for both players $i$ and each $\ell \in\{1, \ldots, k+1\}$, the support of $\lambda_{i}^{\ell}$ is included in $\{w, k+1-\ell\}$ for $\ell \in\left\{1, \ldots, \beta_{j}+1\right\},\{w, 1\}$ for $\ell=\beta_{j}+2,\{w, k+2-\ell\}$ for $\ell \in\left\{\beta_{j}+3, \ldots, k\right\}$, and $\{w, 0\}$ for $\ell=k+1$. Let, for each $\ell \in\{1, \ldots, k+1\}, \lambda_{i}^{\ell}$ be determined by $u_{i}\left(w, \lambda_{i}^{\ell}\right)=$ $u_{i}\left(k-1, \lambda_{i}^{\ell}\right)$. This means that

$$
\begin{array}{ll}
\lambda_{i}^{1}(w)=0 & \lambda_{i}^{1}(k)=1 \\
\lambda_{i}^{2}(w)=\frac{1}{\beta_{j}} & \lambda_{i}^{2}(k-1)=\frac{\beta_{j}-1}{\beta_{j}} \\
\lambda_{i}^{3}(w)=\frac{2}{\beta_{j}+1} & \lambda_{i}^{3}(k-2)=\frac{\beta_{j}-1}{\beta_{j}+1} \\
\ldots & \cdots \\
\lambda_{i}^{\beta_{j}+1}(w)=\frac{\beta_{j}}{2 \beta_{j}-1} & \lambda_{i}^{\beta_{j}+1}\left(\beta_{i}\right)=\frac{\beta_{j}-1}{2 \beta_{j}-1} \\
\lambda_{i}^{\beta_{j}+2}(w)=0 & \lambda_{i}^{\beta_{j}+2}(1)=1 \\
\lambda_{i}^{\beta_{j}+3}(w)=\frac{\beta_{j}+1}{2 \beta_{j}} & \lambda_{i}^{\beta_{j}+3}\left(\beta_{i}-1\right)=\frac{\beta_{j}-1}{2 \beta_{j}} \\
\ldots & \cdots \\
\lambda_{i}^{k}(w)=\frac{k-2}{\beta_{j}+k-3} & \lambda_{i}^{k}(2)=\frac{\beta_{j}-1}{\beta_{j}+k-3} \\
\lambda_{i}^{k+1}(w)=\frac{1}{\beta_{j}} & \lambda_{i}^{k+1}(0)=\frac{\beta_{j}-1}{\beta_{j}}
\end{array}
$$


The LPSs $\lambda_{1}$ and $\lambda_{2}$ determine the following likelihood orderings:

$$
\begin{aligned}
& L_{1}=\left\{\{k\},\{w, k-1\},\{k-2\}, \ldots,\left\{\beta_{1}+1\right\},\left\{\beta_{1}\right\},\{1\},\left\{\beta_{1}-1\right\}, \ldots,\{2\},\{0\}\right\}, \\
& L_{2}=\left\{\{k\},\{w, k-1\},\{k-2\}, \ldots,\left\{\beta_{2}+1\right\},\left\{\beta_{2}\right\},\{1\},\left\{\beta_{2}-1\right\}, \ldots,\{2\},\{0\}\right\} .
\end{aligned}
$$

It can be checked that $L_{1}$ respects the preference restrictions that $u_{2}$ and $\lambda_{2}$ give rise to, and $L_{2}$ respects the preference restrictions that $u_{1}$ and $\lambda_{1}$ give rise to. To see this in the case of $L_{1}$ (the demonstration for $L_{2}$ is symmetric), note:

(a) Player 2 ranks the commitment strategies $0,2,3, \ldots, k$ according to size since $u_{2}\left(s_{2}, \lambda_{2}^{1}\right)=0$ and $u_{2}\left(s_{2}, \lambda_{2}^{2}\right)=s_{2} / \beta_{1}$ for $s_{2} \in 0,2,3, \ldots, k$.

(b) Player 2 is indifferent between the commitment strategy $k-1$ and waiting $w$ since, by construction, $u_{2}\left(w, \lambda_{2}^{\ell}\right)=u_{2}\left(k-1, \lambda_{2}^{\ell}\right)$ for all $\ell \in\{1, \ldots, k+1\}$.

(c) Player 2 ranks the commitment strategy 1 between the commitment strategies $\beta_{1}$ and $\beta_{1}-1$ since

$$
\begin{aligned}
& u_{2}\left(\beta_{1}, \lambda_{2}^{1}\right)=u_{2}\left(1, \lambda_{2}^{1}\right)=u_{2}\left(\beta_{1}-1, \lambda_{2}^{1}\right)=0, \\
& u_{2}\left(\beta_{1}, \lambda_{2}^{2}\right)=u_{2}\left(1, \lambda_{2}^{2}\right)=1>u_{2}\left(\beta_{1}-1, \lambda_{2}^{2}\right)=\frac{\beta_{1}-1}{\beta_{1}}, \\
& u_{2}\left(\beta_{1}, \lambda_{2}^{3}\right)=\frac{2 \beta_{1}}{\beta_{1}+1}>u_{2}\left(1, \lambda_{2}^{3}\right)=1,
\end{aligned}
$$

since $\beta_{1}>1$ and $x_{2}(1, k-2)=1$.

It follows from Blume et al.'s (1991b, Proposition 5) characterization that $\left(\lambda_{1}^{1}, \lambda_{2}^{1}\right)$, where $\lambda_{2}^{1}$ is the mixed strategy of player 1 and $\lambda_{1}^{1}$ is the mixed strategy of player 2 , is a proper equilibrium. Note that, for both players $i, \lambda_{i}^{1}(k)=1$.

Part (ii). Let $\ell$ be any player 1 strategy in $\left\{\beta_{1}+1, \beta_{1}+2, \ldots, k-1\right\}$. Consider the LPSs $\lambda_{1}=\left\{\lambda_{1}^{1}, \ldots, \lambda_{1}^{k+1}\right\}$ and $\lambda_{2}=\left\{\lambda_{2}^{1}, \lambda_{2}^{2}\right\}$ defined by

$$
\begin{array}{lll}
\lambda_{1}^{1}(w)=0 & \lambda_{1}^{1}(k)=1 & \lambda_{2}^{1}(w)=\frac{\beta_{2}}{k} \quad \lambda_{2}^{1}(\ell)=1-\frac{\beta_{2}}{k} \\
\lambda_{1}^{2}(w)=0 & \lambda_{1}^{2}(k-\ell)=1 & \lambda_{2}^{2}\left(s_{1}\right)=\frac{1}{k} \text { for all } s_{1} \in S_{1} \backslash\{w, \ell\} \\
\lambda_{1}^{3}(w)=\frac{1}{\ell-\beta_{1}+1} & \lambda_{1}^{3}(k-1)=\frac{\ell-\beta_{1}}{\ell-\beta_{1}+1} & \\
\ldots & \cdots \\
\lambda_{1}^{\ell+1}(w)=\frac{\ell-1}{2 \ell-\beta_{1}-1} & \lambda_{1}^{\ell+1}(k-\ell+1)=\frac{\ell-\beta_{1}}{2 \ell-\beta_{1}-1} \\
\lambda_{1}^{\ell+2}(w)=\frac{1}{\ell-\beta_{1}+1} & \lambda_{1}^{\ell+2}(k-\ell-1)=\frac{\ell-\beta_{1}}{\ell-\beta_{1}+1} \\
\cdots & \cdots \\
\lambda_{1}^{k}(w)=\frac{k-\ell-1}{k-\beta_{1}-1} & \lambda_{1}^{k}(1)=\frac{\ell-\beta_{1}}{k-\beta_{1}-1} \\
\lambda_{1}^{k+1}(w)=\frac{k-\ell}{k-\beta_{1}} & \lambda_{1}^{k+1}(0)=\frac{\ell-\beta_{1}}{k-\beta_{1}},
\end{array}
$$

with, for each level of these LPSs, zero probability assigned to other strategies.

These LPSs imply that player 1 is indifferent between $w$ and $\ell$ and that player 1 prefers each of these strategies to any strategy in $S_{1} \backslash\{w, \ell\}$, and that player 2 prefers $k$ to any strategy in $S_{2} \backslash\{k\}$. To see this, note: 
(a) It follows that player 1 strictly prefers each of $w$ and $\ell$ to any strategy in $S_{1} \backslash\{w, \ell\}$ since $u_{1}\left(s_{1}, \lambda_{1}^{1}\right)=0$ for all $s_{1} \in S_{1}$ and $u_{1}\left(w, \lambda_{1}^{2}\right)=u_{1}\left(\ell, \lambda_{1}^{2}\right)=\ell$, while $u_{1}\left(s_{1}, \lambda_{1}^{2}\right)=s_{1}<\ell$ if $s_{1}$ is a commitment strategy in $\{1,2, \ldots, \ell-1\}$ and $u_{1}\left(s_{1}, \lambda_{1}^{2}\right)=0<\ell$ if $s_{1}$ is a commitment strategy in $\{\ell+1, \ell+2, \ldots, k\}$. It follows that player 1 is indifferent between $w$ and $\ell$ since $\lambda_{1}^{3}, \lambda_{1}^{4}, \ldots, \lambda_{1}^{k+1}$ have been constructed so that $u_{1}\left(w, \lambda_{1}^{m}\right)=$ $u_{1}\left(\ell, \lambda_{1}^{m}\right)$ for each $m \in\{3,4, \ldots, k+1\}$.

(b) It follows that player 2 strictly prefers $k$ to any strategy in $S_{2} \backslash\{k\}$ since $u_{2}\left(k, \lambda_{2}^{1}\right)=\beta_{2}$ and $u_{2}\left(s_{2}, \lambda_{2}^{1}\right)<\beta_{2}$ for all $s_{2} \in S_{2} \backslash\{k\}$.

Since both $\lambda_{1}$ and $\lambda_{2}$ have full support on the set of opponent strategies, it follows from Blume et al.'s (1991b, Proposition 4) characterization that $\left(\lambda_{1}^{1}, \lambda_{2}^{1}\right)$, where $\lambda_{2}^{1}$ is the mixed strategy of player 1 and $\lambda_{1}^{1}$ is the mixed strategy of player 2 , is a perfect equilibrium where player 1 assigns positive probability to both $w$ and $\ell$ and player 2 assigns probability 1 to $k$.

In a simular fashion we can show that, for any player 2 strategy $\ell \in\left\{\beta_{2}+1, \beta_{2}+\right.$ $2, \ldots, k-1\}$, there exists a perfect equilibrium where player 1 assigns probability 1 to $k$ and player 2 assigns positive probability to both $w$ and $\ell$. 


\section{B Tables}

Table 1: The functioning of the algorithms in game $G_{1}$.

Permissibility

$$
\begin{aligned}
\mathcal{L}_{1}^{0} & =\tilde{\mathcal{L}}_{1}^{*} & \mathcal{L}_{2}^{0} & =\tilde{\mathcal{L}}_{2}^{*} \\
\mathcal{L}_{1}^{\infty} & =\tilde{\mathcal{L}}_{1}^{*} & \mathcal{L}_{2}^{\infty} & =\{(\{U\},\{M, D\}),(\{M\},\{U, D\}),(\{U, M\},\{D\})\}
\end{aligned}
$$

Iterated admissibility

$$
\begin{array}{rlrl}
\mathcal{L}_{1}^{0}=\mathcal{L}_{1}^{*} & \mathcal{L}_{2}^{0}= & \mathcal{L}_{2}^{*} \\
\mathcal{L}_{1}^{1}=\mathcal{L}_{1}^{*} & \mathcal{L}_{2}^{1}=\{(\{U, M\},\{D\}),(\{U\},\{M\},\{D\}),(\{M\},\{U\},\{D\})\} \\
\mathcal{L}_{1}^{2}=\{(\{L\},\{R\})\} & \mathcal{L}_{2}^{2}=\{(\{U, M\},\{D\}),(\{U\},\{M\},\{D\}),(\{M\},\{U\},\{D\})\} \\
\mathcal{L}_{1}^{\infty}=\{(\{L\},\{R\})\} & \mathcal{L}_{2}^{\infty}=\{(\{U\},\{M\},\{D\})\} \\
\text { Proper rationalizability } & & \\
\mathcal{L}_{1}^{0}=\mathcal{L}_{1}^{*} & \mathcal{L}_{2}^{0}=\mathcal{L}_{2}^{*} \\
\mathcal{L}_{1}^{\infty}=\mathcal{L}_{1}^{*} & \mathcal{L}_{2}^{\infty}=\{(\{U\},\{M, D\}),(\{U, M\},\{D\}), \\
& & & (\{U\},\{M\},\{D\}),(\{U\},\{D\},\{M\}),(\{M\},\{U\},\{D\})\}
\end{array}
$$

Full permissibility

$$
\begin{array}{rlrl}
\mathcal{L}_{1}^{0} & =\tilde{\mathcal{L}}_{1}^{*} & \mathcal{L}_{2}^{0} & =\tilde{\mathcal{L}}_{2}^{*} \\
\mathcal{L}_{1}^{1} & =\tilde{\mathcal{L}}_{1}^{*} & \mathcal{L}_{2}^{1} & =\{(\{U\},\{M, D\}),(\{M\},\{U, D\}),(\{U, M\},\{D\})\} \\
\mathcal{L}_{1}^{2} & =\left\{S_{2},(\{L\},\{R\})\right\} & \mathcal{L}_{2}^{2}=\{(\{U\},\{M, D\}),(\{M\},\{U, D\}),(\{U, M\},\{D\})\} \\
\mathcal{L}_{1}^{\infty} & =\left\{S_{2},(\{L\},\{R\})\right\} & \mathcal{L}_{2}^{\infty} & =\{(\{U\},\{M, D\}),(\{U, M\},\{D\})\}
\end{array}
$$


Table 2: The functioning of the algorithms in game $G_{2}$.

$$
\begin{array}{rlrl}
\text { Permissibility } & & \\
\mathcal{L}_{1}^{0}=\tilde{\mathcal{L}}_{1}^{*} & \mathcal{L}_{2}^{0}=\tilde{\mathcal{L}}_{2}^{*} \\
\mathcal{L}_{1}^{\infty}=\tilde{\mathcal{L}}_{1}^{*} & \mathcal{L}_{2}^{\infty}=\{(\{U\},\{M, D\}),(\{M\},\{U, D\}),(\{U, M\},\{D\})\} \\
\text { Iterated admissibility } & & \\
\mathcal{L}_{1}^{0}=\mathcal{L}_{1}^{*} & \mathcal{L}_{2}^{0}=\mathcal{L}_{2}^{*} \\
\mathcal{L}_{1}^{1}=\mathcal{L}_{1}^{*} & \mathcal{L}_{2}^{1}=\{(\{U, M\},\{D\}),(\{U\},\{M\},\{D\}),(\{M\},\{U\},\{D\})\} \\
\mathcal{L}_{1}^{2}=\{(\{L\},\{R\})\} & \mathcal{L}_{2}^{2}=\{(\{U, M\},\{D\}),(\{U\},\{M\},\{D\}),(\{M\},\{U\},\{D\})\} \\
\mathcal{L}_{1}^{\infty}=\{(\{L\},\{R\})\} & \mathcal{L}_{2}^{\infty}=\{(\{U\},\{M\},\{D\})\} \\
\text { Proper rationalizability } & & \\
\mathcal{L}_{1}^{0}=\mathcal{L}_{1}^{*} & \mathcal{L}_{2}^{0}=\mathcal{L}_{2}^{*} \\
\mathcal{L}_{1}^{1}=\mathcal{L}_{1}^{*} & \mathcal{L}_{2}^{1}=\{(\{U\},\{M, D\}),(\{U, M\},\{D\}), \\
& (\{U\},\{M\},\{D\}),(\{U\},\{D\},\{M\}),(\{M\},\{U\},\{D\})\} \\
\mathcal{L}_{1}^{2}=\{(\{L\},\{R\})\} & \mathcal{L}_{2}^{2}=\{(\{U\},\{M, D\}),(\{U, M\},\{D\}), \\
& (\{U\},\{M\},\{D\}),(\{U\},\{D\},\{M\}),(\{M\},\{U\},\{D\})\} \\
\mathcal{L}_{1}^{\infty}=\{(\{L\},\{R\})\} & \mathcal{L}_{2}^{\infty}=\{(\{U\},\{D\},\{M\})\} \\
\text { Full permissibility } & & \\
\mathcal{L}_{1}^{0}=\tilde{\mathcal{L}}_{1}^{*} & \mathcal{L}_{2}^{0}=\tilde{\mathcal{L}}_{2}^{*} \\
\mathcal{L}_{1}^{1}=\tilde{\mathcal{L}}_{1}^{*} & \mathcal{L}_{2}^{1}=\{(\{U\},\{M, D\}),(\{M\},\{U, D\}),(\{U, M\},\{D\})\} \\
\mathcal{L}_{1}^{2}=\left\{S_{2},(\{L\},\{R\})\right\} & \mathcal{L}_{2}^{2}=\{(\{U\},\{M, D\}),(\{M\},\{U, D\}),(\{U, M\},\{D\})\} \\
\mathcal{L}_{1}^{3}=\left\{S_{2},(\{L\},\{R\})\right\} & \mathcal{L}_{2}^{3}=\{(\{U\},\{M, D\}),(\{U, M\},\{D\})\} \\
\mathcal{L}_{1}^{4}=\{(\{L\},\{R\})\} & \mathcal{L}_{2}^{4}=\{(\{U\},\{M, D\}),(\{U, M\},\{D\})\} \\
\mathcal{L}_{1}^{\infty}=\{(\{L\},\{R\})\} & \mathcal{L}_{2}^{\infty}=\{(\{U\},\{M, D\})\} \\
& &
\end{array}
$$


Table 3: The functioning of the algorithms in game $G_{3}$.

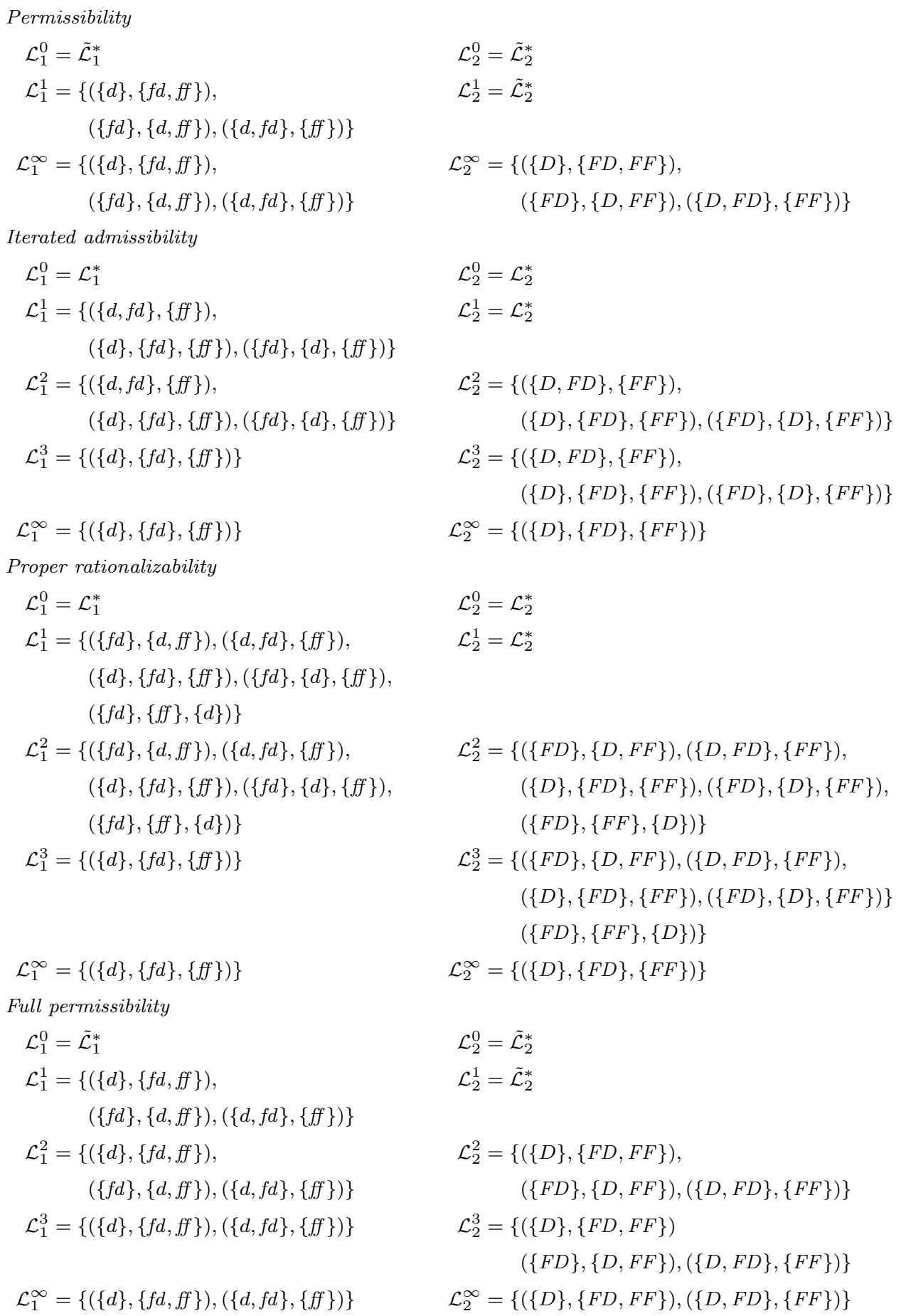


Table 4: The functioning of the algorithms in game $G_{4}$.

\section{Permissibility}

$$
\begin{aligned}
\mathcal{L}_{1}^{0} & =\tilde{\mathcal{L}}_{1}^{*} & \mathcal{L}_{2}^{0} & =\tilde{\mathcal{L}}_{2}^{*} \\
\mathcal{L}_{1}^{\infty} & =\tilde{\mathcal{L}}_{1}^{*} & \mathcal{L}_{2}^{\infty} & =\{(\{U\},\{M, D\}),(\{D\},\{U, M\}),(\{U, D\},\{M\})\}
\end{aligned}
$$

Iterated admissibility

$$
\begin{array}{rlrl}
\mathcal{L}_{1}^{0}=\mathcal{L}_{1}^{*} & \mathcal{L}_{2}^{0}= & \mathcal{L}_{2}^{*} \\
\mathcal{L}_{1}^{1}=\mathcal{L}_{1}^{*} & \mathcal{L}_{2}^{1}=\{(\{U, D\},\{M\}),(\{U\},\{D\},\{M\}),(\{D\},\{U\},\{M\})\} \\
\mathcal{L}_{1}^{2}=\{(\{L\},\{R\})\} & \mathcal{L}_{2}^{2}=\{(\{U, D\},\{M\}),(\{U\},\{D\},\{M\}),(\{D\},\{U\},\{M\})\} \\
\mathcal{L}_{1}^{\infty}=\{(\{L\},\{R\})\} & \mathcal{L}_{2}^{\infty}=\{(\{U\},\{D\},\{M\})\} \\
\text { Proper rationalizability } & & \\
\mathcal{L}_{1}^{0}=\mathcal{L}_{1}^{*} & \mathcal{L}_{2}^{0}=\mathcal{L}_{2}^{*} \\
\mathcal{L}_{1}^{\infty}=\mathcal{L}_{1}^{*} & \mathcal{L}_{2}^{\infty}=\{(\{D\},\{U, M\}),(\{U, D\},\{M\}), \\
& & & (\{U\},\{D\},\{M\}),(\{D\},\{U\},\{M\}),(\{D\},\{M\},\{U\})\}
\end{array}
$$

Full permissibility

$$
\begin{array}{rlrl}
\mathcal{L}_{1}^{0} & =\tilde{\mathcal{L}}_{1}^{*} & \mathcal{L}_{2}^{0} & =\tilde{\mathcal{L}}_{2}^{*} \\
\mathcal{L}_{1}^{1} & =\tilde{\mathcal{L}}_{1}^{*} & \mathcal{L}_{2}^{1} & =\{(\{U\},\{M, D\}),(\{D\},\{U, M\}),(\{U, D\},\{M\})\} \\
\mathcal{L}_{1}^{2} & =\left\{S_{2},(\{L\},\{R\})\right\} & \mathcal{L}_{2}^{2}=\{(\{U\},\{M, D\}),(\{D\},\{U, M\}),(\{U, D\},\{M\})\} \\
\mathcal{L}_{1}^{3} & =\left\{S_{2},(\{L\},\{R\})\right\} & \mathcal{L}_{2}^{3}=\{(\{U\},\{M, D\}),(\{U, D\},\{M\})\} \\
\mathcal{L}_{1}^{4}=\{(\{L\},\{R\})\} & \mathcal{L}_{2}^{4}=\{(\{U\},\{M, D\}),(\{U, D\},\{M\})\} \\
\mathcal{L}_{1}^{\infty}=\{(\{L\},\{R\})\} & \mathcal{L}_{2}^{\infty}=\{(\{U\},\{M, D\})\}
\end{array}
$$

